\title{
Measure data problems for a class of elliptic equations with mixed absorption-reaction
}

\author{
Marie-Françoise Bidaut-Véron* \\ Marta Garcia-Huidobro ${ }^{\dagger}$ \\ Laurent Véron ${ }^{\ddagger}$
}

\begin{abstract}
We study the existence of nonnegative solutions to the Dirichlet problem $\mathcal{L}_{p, q}^{M} u:=-\Delta u+u^{p}-M|\nabla u|^{q}=\mu$ in a domain $\Omega \subset \mathbb{R}^{N}$ where $\mu$ is a nonnegative Radon measure, when $p>1, q>1$ and $M \geq 0$. We also give conditions under which nonnegative solutions of $\mathcal{L}_{p, q}^{M} u=0$ in $\Omega \backslash K$ where $K$ is a compact subset of $\Omega$ can be extended as a solution of the same equation in $\Omega$.

2010 Mathematics Subject Classification: 35J62-35J66-31C15-28A12

Keywords: Elliptic equations, singularities, Bessel capacities, Riesz potential, maximal functions.
\end{abstract}

\section{Contents}

1 Introduction

2 Removable singularities 5

2.1 A priori estimates . . . . . . . . . . . . . . 5

2.2 Proof of Theorem 1.1. . . . . . . . . . . . . . . . . 10

2.3 Proof of Theorem $1.2 \ldots \ldots \ldots \ldots \ldots$

3 Measure data $\quad 16$

3.1 Proof of Theorem 1.3 , the case $1<q<\frac{N}{N-1} \ldots \ldots \ldots$

3.2 Proof of Theorem 1.3 the general case . . . . . . . . . . 20

3.3 Proof of the Corollaries . . . . . . . . . . . . . 23

*Laboratoire de Mathématiques et Physique Théorique, Université de Tours, 37200 Tours, France. E-mail: veronmf@univ-tours.fr

${ }^{\dagger}$ Departamento de Matemáticas, Pontifica Universidad Católica de Chile, Casilla 307, Correo 2, Santiago de Chile. E-mail: mgarcia@mat.uc.cl

${ }^{\ddagger}$ Laboratoire de Mathématiques et Physique Théorique, Université de Tours, 37200 Tours, France. E-mail: veronl@univ-tours.fr 


\section{Introduction}

Let $\Omega$ be a bounded domain of $\mathbb{R}^{N}, N \geq 2$, and $\mathcal{L}_{p, q}^{M}$ be the operator

$$
u \mapsto \mathcal{L}_{p, q}^{M} u:=-\Delta u+|u|^{p-1} u-M|\nabla u|^{q} \quad \text { for all } u \in C^{2}(\Omega)
$$

where $M \geq 0$ and $p, q>1$. We first provide an a priori estimate for a positive solution of (1.1) and its gradient in the range $1<q<p$. Then we study under what conditions on the parameters any solution of

$$
\mathcal{L}_{p, q}^{M} u=0 \quad \text { in } \Omega \backslash K,
$$

where $K$ is a compact subset of $\Omega$, can be extended as a solution of the same equation in whole $\Omega$, and if it is the case, whether the solution is bounded or not in $\Omega$. We also consider the Dirichlet problem with measure data

$$
\begin{aligned}
\mathcal{L}_{p, q}^{M} u=\mu & & \text { in } \Omega \\
u=0 & & \text { in } \partial \Omega,
\end{aligned}
$$

where $\mu$ is a nonnegative bounded Radon measure in $\Omega$ and exhibit conditions which guarantee the existence of nonnegative solutions to this problem.

If $M=0, \mathcal{L}_{p, q}^{M}$ reduces to the Emden-Fowler operator

$$
u \mapsto \mathcal{L}_{p} u:=-\Delta u+|u|^{p-1} u .
$$

Singularity problems for solutions of $\mathcal{L}_{p} u=0$ have been investigated since fourty years, starting with the work of Brezis and Véron [13] who gave conditions for the removability of an isolated singularity. Later on Baras and Pierre [3] extended the result in 13 to more general removable sets, introducing the good framework. They obtained a necessary and sufficient condition expressed in terms of the Bessel capacities $c a p_{2, p^{\prime}}^{\mathbb{R}^{N}}\left(p^{\prime}=\frac{p}{p-1}\right)$ both for the removability of compact subsets of $\Omega$ and the solvability of the associated Dirichlet problem with measure data. Another class of operator strongly related to $\mathcal{L}_{p, q}^{M}$ is the Riccati operator

$$
u \mapsto \mathcal{R}_{q}^{M} u:=-\Delta u-M|\nabla u|^{q} .
$$

The Dirichlet problem with measure data

$$
\begin{aligned}
-\Delta u-M|\nabla u|^{q} & =\mu & & \text { in } \Omega \\
u & =0 & & \text { on } \partial \Omega
\end{aligned}
$$

has been studied by Maz'ya and Verbitsky [19] and Hansson, Maz'ya and Verbitsky [16] when $q>2$ (and also in $\mathbb{R}^{N}$ when $q>1$ ) and Phuc [23. Their results necessitate an extensive use of Riesz potentials.

When $M>0$ there is a balance between the absorption term $|u|^{p-1} u$ and the source term $M|\nabla u|^{q}$, and this interaction is the origin of many unexpected new effects. In the study of singularity problems the effect of the diffusion can be neglectable compared to the two nonlinear terms. The scale of the two opposed reaction terms depends upon the position of $q$ with respect to $\frac{2 p}{p+1}$. This is due to the fact that if $q=\frac{2 p}{p+1}$, (1.1) is equivariant with respect to the scaling transformation $T_{\ell}$ defined for $\ell>0$ by

$$
T_{\ell}[u](x)=\ell^{\frac{2}{p-1}} u(\ell x)=\ell^{\frac{2-q}{q-1}} u(\ell x) .
$$


If $q<\frac{2 p}{p+1}$, the absorption term is dominant and the behaviour of the singular solutions is modelled by the equation $\mathcal{L}_{p} u=0$ studied in [28. If $q>\frac{2 p}{p+1}$, the diffusion is negligible and the behaviour of the singular solutions is modelled by positive separable solutions of $\mathcal{E}_{p, q}^{M} u=0$ where $\mathcal{E}_{p, q}^{M}$ is an eikonal type operator defined by

$$
\mathcal{E}_{p, q}^{M} u=u^{p}-M|\nabla u|^{q} .
$$

This problem is studied in the forthcoming article [8]. If $q=\frac{2 p}{p+1}$, the coefficient $M>0$ plays a fundamental role in the properties of the set of solutions, in particular for the existence of singular solutions and removable singularities; this is not the case when $q \neq \frac{2 p}{p+1}$ since by an homothety $M$ can be assumed to be equal to 1 .

Brezis and Véron proved in 13 that isolated singularities of solutions of $\mathcal{L}_{p} u=0$ are removable when $p \geq \frac{N}{N-2}$. The removability property has been extended to more general sets using a capacity framework in [3]. Using a change of variable inspired by [6] where boundary singularities of solutions of (1.8) are considered we prove a series of removability results for solutions of

$$
\mathcal{L}_{p, q}^{M} u=0 .
$$

Theorem 1.1 Assume $0 \in \Omega \subset \mathbb{R}^{N}, N \geq 3, M>0, p \geq \frac{N}{N-2}, 1<q \leq \frac{2 p}{p+1}$ and $(p, q) \neq\left(\frac{N}{N-2}, \frac{N}{N-1}\right)$. Then any nonnegative solution $u \in C^{2}(\Omega \backslash\{0\})$ of (1.9) in $\Omega \backslash\{0\}$ belongs to $W_{\text {loc }}^{1, q}(\Omega) \cap L_{\text {loc }}^{p}(\Omega)$, and it can be extended as a weak solution of (1.9) in $\Omega$.

Furthermore, if we assume either

(i) $p \geq \frac{N}{N-2}$ and $1<q<\frac{2 p}{p+1}$, or

(ii) $p>\frac{N}{N-2}, q=\frac{2 p}{p+1}$ and

$$
M<m^{*}:=(p+1)\left(\frac{(N-2) p-N}{2 p}\right)^{\frac{p}{p+1}}
$$

then $u \in C^{2}(\Omega)$.

The existence of radial singular solutions when $(p, q)=\left(\frac{N}{N-2}, \frac{N}{N-1}\right)$ and $M>0$, or when $p>\frac{N}{N-2}, q=\frac{2 p}{p+1}$ and $M \geq m^{*}$ shows the optimality of the statements (see [8]). A series of pointwise a priori estimates concerning $u$ and $\nabla u$ are presented in the first section. They are obtained by a combination of Keller-Osserman type estimates, rescaling techniques and Bernstein method. They play a key role for analyzing the case $p=\frac{N}{N-2}$ in the previous theorem, and will be of fundamental importance in the forthcoming paper [8].

The method introduced in the proof of Theorem 1.1 combined with the result of [3] yields a more general removability result. For such a task we denote by $\operatorname{cap} \mathbb{R}_{k, b}^{N}$ the Bessel capacity relative to $\mathbb{R}^{N}$ with order $k>0$ and power $b \in(1, \infty)$. If $k \in \mathbb{N}^{*}$ it coincides with the Sobolev capacity associated to the space $W^{k, b}\left(\mathbb{R}^{N}\right)$ by Calderon's theorem (see e.g. [1 for a detailed presentation).

Theorem 1.2 Let $p>\frac{N}{N-2}$ and $\frac{N}{N-2}<r<p$. Suppose that one of the following conditions is verified: 
(i) either $q=\frac{2 p}{p+1}$ and

$$
0<M<m^{*}(r):=(p+1)\left(\frac{p-r}{p(r-1)}\right)^{\frac{p}{p+1}}
$$

(ii) or $1<q<\frac{2 p}{p+1}$ and $M>0$.

Then, if $K$ is a compact subset of $\Omega$ such that $\operatorname{cap}_{2, r^{\prime}} \mathbb{R}^{N}(K)=0$, any nonnegative solution $u \in C^{2}(\Omega \backslash K)$ of (1.9) in $\Omega \backslash K$ can be extended to $\Omega$ as a solution still denoted by $u$ in the sense of distributions in $\Omega$. Furthermore, if $r \leq \frac{2 N}{N-2}$, then $u \in C^{2}(\Omega)$.

Next we obtain sufficient conditions on a positive measure in $\Omega$ in order (1.3) be solvable. In the sequel we assume that $\Omega \subset \mathbb{R}^{N}, N \geq 2$, is a bounded smooth domain. We denote by $\mathfrak{M}(\Omega)$ (resp. $\mathfrak{M}^{b}(\Omega)$ ) the set of Radon measures (resp. bounded Radon measures) in $\Omega$ and by $\mathfrak{M}_{+}(\Omega)$ (resp. $\mathfrak{M}_{+}^{b}(\Omega)$ ) its positive cone. The total variation norm of a bounded measure $\mu$ is $\|\mu\|_{\mathfrak{M}}$.

Since for any $\mu \in \mathfrak{M}_{+}^{b}(\Omega)$ the nonnegative solutions of $\mathcal{L}_{p} v=\mu$ and $\mathcal{R}_{q}^{M} w=$ $\mu$ are respectively a subsolution and a supersolution of equation (1.3) and they satisfy $0 \leq v \leq w$, the construction of $v$ and $w$ is the key-stone for solving (1.3). It is known that these two problems can be solved when the measure $\mu$ satisfies some continuity properties with respect to some specific capacities.

Theorem 1.3 Assume $p>1,1<q<2$. Let $\mu \in \mathfrak{M}_{+}^{b}(\Omega)$. If $\mu$ satisfies

$$
\mu(E) \leq C \min \left\{\operatorname{cap}_{2, p^{\prime}}^{\mathbb{R}^{N}}(E), \operatorname{cap}_{1, q^{\prime}}^{\mathbb{R}^{N}}(E)\right\} \quad \text { for all Borel sets } E \subset \Omega,
$$

there is a constant $c_{0}>0$ such that for any $0 \leq c \leq c_{0}$ there exists a function $u \in W_{0}^{1, q}(\Omega) \cap L^{p}(\Omega), u \geq 0$, satisfying

$$
-\int_{\Omega} u \Delta \zeta d x+\int_{\Omega}\left(u^{p}-M|\nabla u|^{q}\right) \zeta d x=c \int_{\Omega} \zeta d \mu \quad \text { for all } \zeta \in C_{c}^{2}(\bar{\Omega}) .
$$

The condition on the measure is satisfied if $W^{-1, q}(\Omega) \hookrightarrow W^{-2, p}(\Omega)$, and we prove the following:

Corollary 1.4 Let $\frac{N p}{N+p} \leq q<2$ and $\mu \in \mathfrak{M}_{+}^{b}(\Omega)$ be such that

$$
\mu(E) \leq C \operatorname{cap} \mathbb{R}_{1, q^{\prime}}^{\mathbb{R}^{N}}(E) \quad \text { for all Borel set } E \subset \Omega,
$$

for some $C>0$, then there exists a constant $c_{1}>0$ such that for any $0 \leq c \leq c_{1}$ there exists a nonnegative function $u \in W_{0}^{1, q}(\Omega) \cap L^{p}(\Omega)$ satisfying (1.13).

By comparison results between capacities we have another type of result:

Corollary 1.5 Let $\frac{N}{N-1} \leq q \leq \frac{2 p}{p+1}$. If $\mu \in \mathfrak{M}_{+}^{b}(\Omega)$ satisfies,

$$
\mu(E) \leq \operatorname{Ccap}_{2, p^{\prime}}^{\mathbb{R}^{N}}(E) \quad \text { for all Borel set } E \subset \Omega,
$$

for some $C>0$, then there exists $c_{2}>0$ such that for any $0 \leq c \leq c_{2}$ there exists a nonnegative function $u \in W_{0}^{1, q}(\Omega) \cap L^{p}(\Omega)$ satisfying (1.13). 
As an application of the previous results, we prove the following

Corollary 1.6 Let $p>1,1<q<2$ and $\mu \in \mathfrak{M}_{+}^{b}(\Omega)$. There exists a function $u \in W_{0}^{1, q}(\Omega) \cap L^{p}(\Omega)$ solution of (1.13) if one of the following conditions is satisfied:

(i) When $p<\frac{N}{N-2}$ and $q<\frac{N}{N-1}$, if $\|\mu\|_{\mathfrak{M}} \leq c_{3}$ for some $c_{3}>0$.

(ii) When $p<\frac{N}{N-2}$ and $\frac{N}{N-1} \leq q<2$, if $\mu$ satisfies (1.14) for some $C>0$. In that case there exists $c_{4}>0$ such that there must hold $0<c<c_{4}$ in problem (1.12).

(iii) When $p \geq \frac{N}{N-2}$ and $q<\frac{N}{N-1}$, if $\|\mu\|_{\mathfrak{M}} \leq c_{4}^{*} M^{-\frac{1}{q-1}}$ for some $c_{4}^{*}=$ $c_{4}^{*}(N, q, \Omega)>0$ which can be estimated, and if

$$
\mu(E)=0 \quad \text { for all Borel set } \subset \Omega \text { such that } \operatorname{cap}_{2, p^{\prime}}^{\mathbb{R}^{N}}(E)=0 .
$$

In the case (i) we show in a forthcoming article 8 and by a completely different method that there is no restriction on $c$ if $\mu=c \delta_{a}$ for some $a \in \Omega$. In the above mentioned article we construct many types of local or global singular solutions using methods inherited from dynamical systems.

Acknowledgements This article has been prepared with the support of the FONDECYT grants 1210241 and 1190102 for the three authors.

\section{Removable singularities}

Throughout this article we denote by $c$ and $C$ generic constants the value of which may vary from one occurrence to another even within a single string of estimates, and by $c_{j},(j=1,2, \ldots)$ some constants which have a more important significance and a more precise dependence with respect to the parameters.

\subsection{A priori estimates}

We give two estimates for positive solutions of (1.1) which differ according to the sign of $M$. If $G$ is an open subset of $\mathbb{R}^{N}$ we set $d_{G}(x)=\operatorname{dist}(x, \partial G)$

Proposition 2.1 Let $G \subset \mathbb{R}^{N}$ be an open subset, $M>0$ and $1<q<p$. If $u \in C^{1}(G)$ is a nonnegative solution of (1.1), there holds,

$$
u(x) \leq c_{5} \max \left\{M^{\frac{1}{p-q}}\left(d_{G}(x)\right)^{-\frac{q}{p-q}},\left(d_{G}(x)\right)^{-\frac{2}{p-1}}\right\} \quad \text { for all } x \in G,
$$

for some $c_{5}=c_{5}(N, p, q)>0$.

Proof. Following the method of Keller [17] and Osserman [22, we fix $x \in G$ and $0<a<d_{G}(x)$, and introduce $U(z)=\lambda\left(a^{2}-|z-x|^{2}\right)^{-b}$ for some $b>0$. Then putting $r=|x-z|$ and $\tilde{U}(r)=U(z)$, we have in $B_{a}(x)$

$$
\begin{gathered}
L \tilde{U}=-\tilde{U}^{\prime \prime}-\frac{N-1}{r} \tilde{U}^{\prime}-M\left|\tilde{U}^{\prime}\right|^{q}+\tilde{U}^{p} \\
=\lambda\left(a^{2}-r^{2}\right)^{-2-b}\left[\lambda^{p-1}\left(a^{2}-r^{2}\right)^{2-b(p-1)}+2 b(N-2(b+1)) r^{2}-2 N b a^{2}\right. \\
\left.\quad-M 2^{q} b^{q} \lambda^{q-1} r^{q}\left(a^{2}-r^{2}\right)^{2+b-q(b+1)}\right] .
\end{gathered}
$$


If $M>0$, the two necessary conditions on $b>0$ to be fulfilled is order that $\tilde{U}$ is a supersolution in $B_{|a|}(a)$ are

$$
\begin{aligned}
& 2-b(p-1) \leq 0 \Longleftrightarrow b(p-1) \geq 2, \\
& 2+b-q(b+1) \geq 2-b(p-1) \Longleftrightarrow b(p-q) \geq q .
\end{aligned}
$$

The above inequalities are satisfied if

$$
b=\max \left\{\frac{2}{p-1}, \frac{q}{p-q}\right\} .
$$

If $q>\frac{2 p}{p+1}$ then $b=\frac{q}{p-q}$ and

$$
L \tilde{U} \geq \lambda\left(a^{2}-r^{2}\right)^{-\frac{2 p-q}{p-q}}\left[\lambda^{q-1}\left(\lambda^{p-q}-M 2^{q} b^{q} \rho^{q}\right)\left(a^{2}-r^{2}\right)^{\frac{2 p-q(p+1)}{p-q}}-(3 b+1) N a^{2}\right] .
$$

There exists $c_{5}^{1}>0$ depending on $N, p$ and $q$ such that if we choose

$$
\lambda=c_{5}^{1} \max \left\{M^{\frac{1}{p-q}} a^{\frac{q}{p-q}}, a^{\frac{2 p(q-1)}{(p-1)(p-q)}}\right\},
$$

there holds

$$
L \tilde{U} \geq 0 \quad \text { in } B_{a}(x) .
$$

Since $\tilde{U}(z) \rightarrow \infty$ when $r \rightarrow a$, we derive by the maximum principle that $u \leq \tilde{U}$ in $B_{a}(x)$. In particular

$$
u(x) \leq \tilde{U}(x)=\lambda a^{-\frac{2 q}{p-q}}=c_{5}^{1} \max \left\{M^{\frac{1}{p-q}} a^{-\frac{q}{p-q}}, a^{-\frac{2}{p-1}}\right\} .
$$

If $q \leq \frac{2 p}{p+1}$ then $b=\frac{2}{p-1}$ and

$$
\begin{aligned}
& L \tilde{U} \geq \lambda\left(|a|^{2}-\rho^{2}\right)^{-\frac{2 p}{p-1}}\left[\lambda^{p-1}+\frac{2}{p-1}\left(N-\frac{2(p+1)}{p-1}\right) \rho^{2}-\frac{2 N}{p-1}|a|^{2}\right.\left.-M 2^{q}\left(\frac{2}{p-1}\right)^{q} \lambda^{q-1} \rho^{q}\left(|a|^{2}-\rho^{2}\right)^{\frac{2 p-q(p+1)}{p-1}}\right] \\
& \geq \lambda\left(|a|^{2}-\rho^{2}\right)^{-\frac{2 p}{p-1}}\left[\lambda^{p-1}-c_{2}|a|^{2}-c_{3} \lambda^{q-1} M|a|^{\frac{4 p-q(p+3)}{p-1}}\right] .
\end{aligned}
$$

Hence, if $q=\frac{2 p}{p+1},(2.3)$ holds if for some $c_{5}^{2}>0$ depending on $N, p, q$,

$$
\lambda=c_{5}^{2} \max \left\{M^{\frac{p+1}{p(p-1)}}, 1\right\}|a|^{\frac{2}{p-1}}
$$

which yields

$$
u(x) \leq \tilde{U}(x)=\lambda a^{-\frac{4}{p-1}}=c_{5}^{2} \max \left\{M^{\frac{p+1}{p(p-1)}}, 1\right\} a^{-\frac{2}{p-1}} .
$$

While if $q<\frac{2 p}{p+1}$, we choose

$$
\lambda=c_{5}^{3} \max \left\{M^{\frac{1}{p-q}} a^{\frac{4 p-q(p+3)}{(p-1)(p-q)}}, a^{\frac{2}{p-1}}\right\},
$$


where $c_{5}^{3}>0=c_{5}^{3}(N, p, q)$, which implies

$$
u(x) \leq \tilde{U}(x)=\lambda a^{-\frac{4}{p-1}}=c_{5}^{3} \max \left\{M^{\frac{1}{p-q}} a^{-\frac{q}{p-q}}, a^{-\frac{2}{p-1}}\right\} .
$$

By letting $a \uparrow d_{G}(x)$ we derive (2.1) with a constant $c_{5}=c_{5}^{3}$, depending on $N, p, q$.

Corollary 2.2 Under the assumptions of Proposition 2.1] with $G=B_{2 R} \backslash\{0\}$, there holds for $x \in B_{R} \backslash\{0\}$,

$$
u(x) \leq c_{5} \max \left\{M^{\frac{1}{p-q}}|x|^{-\frac{q}{p-q}},|x|^{-\frac{2}{p-1}}\right\} .
$$

We infer from Proposition 2.1 an estimate of the gradient of a positive solution when $M>0$. We set $\sigma=2 p-q(p+1)$, then $\sigma>0$ (resp. $\sigma<0$ ) according $2 p>q(p+1)$ (resp. $2 p<q(p+1)$ ).

Proposition 2.3 Let $p>q>1$. For any $M_{0}>0$ and $R>0$ there exists a constant $c_{8}=c_{8}\left(N, p, q, M_{0} R^{\frac{\sigma}{p-1}}\right)$ such that, for $0<M \leq M_{0}$ there holds:

(i) If $q \leq \frac{2 p}{p+1}$ (then $\sigma \geq 0$ ), any positive solution u of (1.1) in $B_{2 R} \backslash\{0\}$ satisfies

$$
|\nabla u(x)| \leq c_{8} \max \left\{M^{\frac{1}{p-q}}|x|^{-\frac{p}{p-q}},|x|^{-\frac{p+1}{p-1}}\right\},
$$

for all $x \in B_{R} \backslash\{0\}$.

(ii) If $\frac{2 p}{p+1} \leq q \leq 2$ (then $\sigma \leq 0$ ), any positive solution $u$ of (1.1) in $\mathbb{R}^{N} \backslash \bar{B}_{\frac{R}{2}}$ satisfies (2.1) for all $x \in \mathbb{R}^{N} \backslash B_{R}$.

Proof. (i) For $0<r<2 R$ we set

$$
u(x)=r^{-\frac{2}{p-1}} u_{r}\left(\frac{x}{r}\right)=r^{-\frac{2}{p-1}} u_{r}(y) \quad \text { with } y=\frac{x}{r} .
$$

If $\frac{r}{2}<|x|<2 r$, then $\frac{1}{2}<|y|<2$ and $u_{r}>0$ satisfies

$$
-\Delta u_{r}+u_{r}^{p}-M r^{\frac{2 p-q(p+1)}{p-1}}\left|\nabla u_{r}\right|^{q}=0 \quad \text { in } B_{2} \backslash B_{\frac{1}{2}} .
$$

Since $0<M r^{\frac{\sigma}{p-1}} \leq M(2 R)^{\frac{\sigma}{p-1}} \leq M_{0}(2 R)^{\frac{\sigma}{p-1}}$ as $\sigma \geq 0$, it follows that

$$
\max \left\{\left|\nabla u_{r}(z)\right|: \frac{2}{3}<|z|<\frac{3}{2}\right\} \leq c \max \left\{\left|u_{r}(z)\right|: \frac{1}{2}<|z|<2\right\},
$$

where $c$ depends on $N, p, q$ and $R^{\frac{\sigma}{p-1}} M_{0}$ (see e.g. [14, Chapter 13]). From Proposition 2.1 there holds

$$
\max \left\{\left|u_{r}(z)\right|: \frac{1}{2}<|z| \leq 2\right\} \leq 2^{\frac{2}{p-1}} c_{5} \max \left\{M^{\frac{1}{p-q}} r^{\frac{2 p-q(p+1)}{(p-1)(p-q)}}, 1\right\}
$$

by (2.1). Therefore

$$
\begin{aligned}
\max \left\{|\nabla u(y)|: \frac{r}{2}<|z|<2 r\right\} & \leq 2^{\frac{2}{p-1}} c c_{5} r^{-\frac{p+1}{p-1}} \max \left\{M^{\frac{1}{p-q}} r^{\frac{2 p-q(p+1)}{(p-1)(p-q)}}, 1\right\} \\
& \leq c_{8} \max \left\{M^{\frac{1}{p-q}}|x|^{-\frac{p}{p-q}},|x|^{-\frac{p+1}{p-1}}\right\}
\end{aligned}
$$

which implies (2.8). 
(ii) For $r>R$ we define $u_{r}$ as in (i). It satisfies (2.9) and since $\sigma \leq 0$, we have again $0<M r^{\frac{\sigma}{p-1}} \leq M R^{\frac{\sigma}{p-1}} \leq M_{0} R^{\frac{\sigma}{p-1}}$ if $r \geq R$. Since $1<q<2$, (2.10) holds and we derive (2.8).

Remark. If $q=\frac{2 p}{p+1}$ the constant $c_{8}$ depends only on $N$ and $p$.

The previous estimate necessitates $1<q \leq 2$. This limitation can be by passed in some cases using the Bernstein approach.

Lemma 2.4 Assume $p, q>1$ and $M>0$. If $u \in C^{2}\left(\bar{B}_{2 R}\right)$ is a nonnegative solution of (1.1) in $B_{2 R}$, there holds

$$
|\nabla u(x)| \leq c_{9}\left(|x|^{-\frac{1}{q-1}}+\max _{\mid z-x] \leq \frac{|x|}{2}} u^{\frac{p}{q}}(z)\right) \quad \text { for all } x \in B_{\frac{R}{2}},
$$

$c_{9}>0$ depends on $N, p, q$ and $M$.

Proof. Set $z=|\nabla u|^{2}$, then by a classical computation and the use of Schwarz inequality,

$$
-\Delta|\nabla u|^{2}+\frac{1}{N}(\Delta u)^{2}+\langle\nabla \Delta u, \nabla u\rangle \leq 0 .
$$

Replacing $\Delta u$ by its expression from $\mathcal{L}_{p, q}^{M} u=0$, we obtain

$$
-\Delta z+\frac{2}{N}\left(u^{2 p}+M^{2} z^{q}-2 M u^{p} z^{\frac{q}{2}}\right)+2 p u^{p-1} z \leq q M z^{\frac{q}{2}-1}\langle\nabla z, \nabla u\rangle .
$$

We notice that

$$
q M z^{\frac{q}{2}-1}\langle\nabla z, \nabla u\rangle \leq q M z^{\frac{q}{2}-1}|\nabla z| \sqrt{z}=q M z^{\frac{q}{2}} \frac{|\nabla z|}{\sqrt{z}} \leq \frac{M^{2} z^{q}}{2 N}+\frac{2 N q^{2}}{M^{2}} \frac{|\nabla z|^{2}}{z},
$$

and

$$
\frac{4 M}{N} u^{p} z^{\frac{q}{2}} \leq \frac{M^{2} z^{q}}{2 N}+\frac{8 u^{2 p}}{N M^{2}}
$$

thus

$$
-\Delta z+\frac{M^{2} z^{q}}{N} \leq \frac{2 N q^{2}}{M^{2}} \frac{|\nabla z|^{2}}{z}+\frac{2}{N}\left(\frac{4}{M^{2}}-1\right) u^{2 p} .
$$

For simplicity we set

$$
A=\frac{M^{2}}{N}, B=\frac{2 N q^{2}}{M^{2}} \text { and } C=\frac{2}{N}\left(\frac{4}{M^{2}}-1\right)_{+|z-x| \leq \frac{|x|}{2}} u^{2 p}(z)
$$

Then $z$ satisfies

$$
-\Delta z+A z^{q} \leq B \frac{|\nabla z|^{2}}{z}+C \quad \text { in } B_{\frac{R}{2}}(x)
$$

and obtain by [5] Lemma 3.1] (see also a simpler approach in [7, Lemma 2.2]),

$$
z(x) \leq c_{10}\left(|x|^{-\frac{2}{q-1}}+C^{\frac{1}{q}}\right)
$$

where $c_{10}>0$ depends on $N, p, q$ and $M$. This yields (2.12).

Remark. The constants $c_{9}$ and $c_{10}$ can be expressed in terms of $M$, but their stability when $M \rightarrow 0$ is not clear since in the limit case of the equation $\mathcal{L}_{p} u=0$ the estimate of the gradient obtained by a very different and much simpler method combining the Keller-Osserman estimate and scaling methods.

Using Corollary 2.2 we obtain the new estimate 
Corollary 2.5 Assume $1<q<p$ and $M>0$. Then any nonnegative solution $u \in C^{2}\left(B_{2 R}\right)$ of (1.1) satisfies

$$
|\nabla u(x)| \leq c_{11}\left(|x|^{-\frac{1}{q-1}}+\max \left\{M^{\frac{p}{q(p-q}}|x|^{-\frac{p}{p-q}},|x|^{-\frac{2 p}{q(p-1)}}\right\}\right) \quad \text { for all } x \in B_{\frac{R}{2}},
$$

where $c_{11}>0$ depends on $N, p, q$ and $M$.

Then we can combine this estimate with Proposition 2.1 to complete the cases not treated in Proposition 2.3

Proposition 2.6 Let $1<q<p$. For any $M>0$ there exists a constant $c_{12}=c_{12}(N, p, q, M)>0$ such that:

(i) If $\frac{2 p}{p+1} \leq q<p$, any positive solution $u$ of (1.1) in $B_{2 R} \backslash\{0\}$ with $0<R \leq 1$ satisfies,

$$
|\nabla u(x)| \leq c_{12} \max \left\{M^{\frac{p}{q(p-q)}}|x|^{-\frac{p}{p-q}},|x|^{-\frac{2 p}{q(p-1)}}\right\} \quad \text { for all } x \in B_{R} \backslash\{0\} .
$$

(ii) If $1<q \leq \frac{2 p}{p+1}$, any positive solution $u$ of (1.1) in $\mathbb{R}^{N} \backslash \bar{B}_{\frac{R}{2}}$ with $R \geq 1$ satisfies,

$$
|\nabla u(x)| \leq c_{12} \max \left\{M^{\frac{p}{q(p-q)}}|x|^{-\frac{p}{p-q}},|x|^{-\frac{1}{q-1}}\right\} \quad \text { for all } x \in \mathbb{R}^{N} \backslash B_{R} .
$$

Proof. We can compare the different exponents of $|x|$ which appear in the expressions (2.8) and (2.14)

$$
\begin{array}{ll}
\frac{p}{p-q}<\frac{p+1}{p-1}<\frac{2 p}{q(p-1)}<\frac{1}{q-1} & \text { if } 1<q<\frac{2 p}{p+1}, \\
\frac{1}{q-1}<\frac{2 p}{q(p-1)}<\frac{p+1}{p-1}<\frac{p}{p-q} & \text { if } \frac{2 p}{p+1}<q<p,
\end{array}
$$

with equality if $q=\frac{2 p}{p+1}$. If $1<q \leq \frac{2 p}{p+1}$ (resp. $\frac{2 p}{p+1} \leq \leq 2$ ), estimate (2.8) is better than (2.14) in $B_{R} \backslash\{0\}$ (resp. $\mathbb{R}^{N} \backslash B_{2 R}$ ). Then (2.15) and (2.16) follow from (2.14) and (2.17).

In the case $M<0$ an upper estimate on a solution is obtained by combining a result of Lions and the method of Keller and Osserman.

Proposition 2.7 Let $G \subset \mathbb{R}^{N}$ be an open subset, $M \leq 0$ and $p, q>1$. If $u \in C^{1}(G)$ is a nonnegative solution of $\mathcal{L}_{p, q}^{M} u=0$, there exists $c_{6}=c_{6}(N, p)>0$, $c_{7}=c_{7}(N, q)>0$ and $\delta=\delta(G)>0$ such that there holds for all $x \in G$ and $\varnothing<\delta \leq \delta(G)$,

$$
u(x) \leq \min \left\{c_{6}\left(d_{G}(x)\right)^{-\frac{2}{p-1}}, c_{7}|M|^{-\frac{1}{q-1}}\left(d_{G}(x)\right)^{-\frac{2-q}{q-1}}+\max _{d_{G}(z)=\delta} u(z),\right\} .
$$

Proof. This estimates follows from the fact that the solutions of $\mathcal{L}_{p, q}^{M} u=0$ are subsolutions of $\mathcal{L}_{p} u=0$ and $\mathcal{R}_{q}^{M} u=0$. The estimate $u(x) \leq c_{6}\left(d_{G}(x)\right)^{-\frac{2}{p-1}}$ corresponds to the Keller-Osserman estimate for solutions of $\mathcal{L}_{p} u=0$. The second estimate corresponds to the fact that if $u$ is a positive solution of $\mathcal{R}_{q}^{M} u=$ 0 in $G$ there holds (see [18, Theorem IV-1])

$$
|\nabla u(x)| \leq c_{7}^{\prime}|M|^{-\frac{1}{q-1}}\left(d_{G}(x)\right)^{-\frac{1}{q-1}} .
$$


Integrating this inequality yields the second part of the inequality.

Remark. This estimate can be transformed into the universal estimate

$$
u(x) \leq \min \left\{c_{6}\left(d_{G}(x)\right)^{-\frac{2}{p-1}}, c_{7}|M|^{-\frac{1}{q-1}}\left(d_{G}(x)\right)^{-\frac{2-q}{q-1}}+c_{6} \delta^{-\frac{2}{p-1}},\right\},
$$

since $\max _{d_{G}(z)=\delta} u(z) \leq c_{6} \delta^{-\frac{2}{p-1}}$ by (2.18).

The gradient estimates are due to Nguyen [20, Proposition 1.1]. Below we recall his result proved by the Bernstein method in a more general framework but which can also be obtained by scaling techniques in the present case.

Proposition 2.8 Let $p>1$ and $1<q<2$. For any $M<0$ and $R>0$ there exists a constant $c_{12}^{\prime}=c_{12}^{\prime}(N, p, q, M)>$,0 such that: if $u$ is a positive solution of (1.1) in $B_{2 R} \backslash\{0\}$, there holds

$$
u(x)+|x||\nabla u(x)| \leq c_{12}^{\prime} \max \left\{|x|^{-\frac{2}{p-1}},|x|^{-\frac{2-q}{q-1}}\right\} \quad \text { for all } x \in B_{R} \backslash\{0\} .
$$

Remark. There are many estimates of positive solutions of (1.1) (or even with $u^{p}$ replaced by $f(u)$ ) in a domain which tends to infinity on the boundary (large solutions) or of solutions in $\mathbb{R}^{N}$ (ground states). Many of these estimates are obtained by comparison with one dimensional problems and they can be found in [2], 4], [15].

\subsection{Proof of Theorem 1.1}

Without loss of generality we can assume that $u \in C^{2}\left(\bar{\Omega} \backslash\{0\}\right.$ and $\bar{B}_{2 R_{0}} \subset \Omega$ with $2 R_{0} \leq 1$. If $M \leq 0, u$ is a nonnegative subsolution of $-\Delta u+u^{p}=0$, hence it is bounded in $\bar{\Omega}$ by 13 .

Step 1. We assume $M>0$ and we prove first that under condition (i) or (ii), $|\nabla u|^{q} \in L^{1}(\Omega), u \in L^{p}(\Omega)$, and then

$$
\int_{\Omega}\left(-u \Delta \zeta+u^{p} \zeta-M|\nabla u|^{q} \zeta\right) d x=0 \quad \forall \zeta \in W^{2, \infty}(\Omega) \cap C_{c}^{1}(\bar{\Omega}) .
$$

By Proposition 2.3

$$
|\nabla u|^{q} \leq c|x|^{-\frac{(p+1) q}{p-1}} \quad \text { in } B_{R_{0}},
$$

since $q \leq \frac{2 p}{p+1}$, and where $c$ depends also on $M$. By (i) or (ii), $\frac{(p+1) q}{p-1}<N$. Hence $\nabla u \in L_{\text {loc }}^{q}(\Omega)$.

For any $\epsilon>0$ small enough we denote by $\rho_{\epsilon}$ a nonnegative $C^{\infty}$-function such that $\operatorname{supp}\left(\rho_{\epsilon}\right) \subset \bar{B}_{\epsilon}, 0 \leq \rho_{\epsilon} \leq 1,\left|\nabla \rho_{\epsilon}\right| \leq 2 \epsilon^{-1} \chi_{\bar{B}_{\epsilon}}$ and we set $\eta_{\epsilon}=1-\rho_{\epsilon}$. Then

$$
-\int_{B_{2 R_{0}}}\left\langle\nabla u, \nabla \rho_{\epsilon}\right\rangle d x+\int_{B_{2 R_{0}}} u^{p} \eta_{\epsilon} d x+\int_{\partial B_{2 R_{0}}} \frac{\partial u}{\partial \mathbf{n}} d S=M \int_{B_{2 R_{0}}}|\nabla u|^{q} \eta_{\epsilon} d x .
$$

Next

$$
\left|\int_{B_{2 R_{0}}}\left\langle\nabla u, \nabla \rho_{\epsilon}\right\rangle d x\right| \leq 2 c_{N} \epsilon^{\frac{N}{q^{\prime}}-1}\left(\int_{B_{\epsilon}}|\nabla u|^{q} d x\right)^{\frac{1}{q}} \rightarrow 0 \quad \text { as } \epsilon \rightarrow 0,
$$


since $1<q \leq \frac{N}{N-1}$. Since $|\nabla u|^{q} \in L^{1}\left(B_{2 R_{0}}\right)$ we deduce by monotone convergence that $u^{p} \in L^{1}\left(B_{2 R_{0}}\right)$. Finally, if $\zeta \in C_{0}^{\infty}(\Omega)$ and $\zeta_{\epsilon}=\zeta \eta_{\epsilon}$, we have

$$
\int_{\Omega}\left\langle\nabla u, \nabla \zeta_{\epsilon}\right\rangle d x+\int_{\Omega} u^{p} \zeta_{\epsilon} d x-M \int_{\Omega}|\nabla u|^{q} \zeta_{\epsilon} d x=0 .
$$

Letting $\epsilon \rightarrow 0$ and using (2.23), we infer that $u$ satisfies

$$
\int_{\Omega}\langle\nabla u, \nabla \zeta\rangle d x+\int_{\Omega} u^{p} \zeta d x-M \int_{\Omega}|\nabla u|^{q} \zeta d x=0 .
$$

Hence it is a weak solution of (1.9) in $\Omega$.

Step 2. Let us assume that $p>\frac{N}{N-2}$. If $u$ is nonnegative and not identically zero, it is positive in $\Omega \backslash\{0\}$ by the maximum principle. We set $u=v^{b}$ with $0<b \leq 1$. Then

$$
-\Delta v-(b-1) \frac{|\nabla v|^{2}}{v}+\frac{1}{b} v^{1+(p-1) b}-M b^{q-1} v^{(b-1)(q-1)}|\nabla v|^{q}=0 .
$$

For $\epsilon>0$,

$$
v^{(b-1)(q-1)}|\nabla v|^{q} \leq \frac{q \epsilon^{\frac{2}{q}}}{2} \frac{|\nabla v|^{2}}{v}+\frac{2-q}{2 \epsilon^{\frac{2}{2-q}}} v^{1+\frac{2 b(q-1)}{2-q}} .
$$

Therefore

$$
-\Delta v+\left(1-b-M \frac{q b^{q-1} \epsilon^{\frac{2}{q}}}{2}\right) \frac{|\nabla v|^{2}}{v}+\frac{1}{b} v^{1+b(p-1)}-M b^{q-1} \frac{2-q}{2 \epsilon^{\frac{2}{2-q}}} v^{1+\frac{2 b(q-1)}{2-q}}=0 .
$$

We notice that $1+\frac{2 b(q-1)}{2-q}=1+b(p-1)-a$ with $a=b \frac{2 p-(p+1) q}{2-q} \geq 0$. We fix $b$ as follows,

$$
(p-1) b+1=\frac{N}{N-2} \Longleftrightarrow b=\frac{2}{(N-2)(p-1)},
$$

hence $p>\frac{N}{N-2}$ if and only if $0<b<1$. Next we impose

$$
1-b-M \frac{q b^{q-1} \epsilon^{\frac{2}{q}}}{2}=0 \Longleftrightarrow \epsilon=\left(\frac{2(1-b)}{M q b^{q-1}}\right)^{\frac{q}{2}}=\left(\frac{2((N-2) p-N)}{M q b^{q-1}(N-1)(p-1)}\right)^{\frac{q}{2}} .
$$

This transforms (2.25) into

$$
-\Delta v+\frac{(N-2)(p-1)}{2} v^{\frac{N}{N-2}}-\frac{(2-q) b^{q-1}}{2}\left(\frac{q}{2(1-b)}\right)^{\frac{q}{2-q}} M^{\frac{2}{2-q}} v^{\frac{N}{N-2}-a} \leq 0 .
$$

We first assume that $0<q<\frac{2 p}{p+1}$. Then $a>0$, hence there exists $A>0$, depending on $M$, such that

$$
-\Delta v+\frac{(N-2)(p-1)}{4} v^{\frac{N}{N-2}} \leq A .
$$

Set $\tilde{v}=\left(v-c A^{\frac{N-2}{N}}\right)_{+}^{\frac{N}{N-2}}$ with $c=\left(\frac{4}{(N-2)(p-1)}\right)^{\frac{N}{N-2}}$ satisfies

$$
-\Delta \tilde{v}+\frac{(N-2)(p-1)}{4} \tilde{v}^{\frac{N}{N-2}} \leq 0
$$


By [13], $\tilde{v} \leq \max _{\partial \Omega} \tilde{v}$ which implies $v \leq c A^{\frac{N-2}{N}}+\max _{\partial \Omega} v$ and therefore $u(x) \leq B$ for some $B \geq 0$ in $\Omega$. Furthermore $|\nabla u|^{q-1} \in L^{\frac{q}{q-1}}(\Omega)$ since $\nabla u \in L^{q}(\Omega)$, and $\frac{q}{q-1}>N$ as we assume $q<\frac{N}{N-1}$. Writing (1.9) under the form

$$
-\Delta u+u^{p}-M C(x)|\nabla u|=0,
$$

with $C(x)=|\nabla u(x)|^{q-1}$, it follows from Serrin's theorem [25. Theorem 10] that the singularity at 0 is removable and $u$ can be extended as a regular solution of (1.9) in $\Omega$. Hence $u \in C^{2}(\Omega)$.

Then we assume that $q=\frac{2 p}{p+1}$. By the choice of $b$ in (2.26), inequality (2.25) becomes

$$
-\Delta v+\left(1-b-\frac{M p b^{\frac{p-1}{p+1}} \epsilon^{\frac{p+1}{p}}}{p+1}\right) \frac{|\nabla v|^{2}}{v}+\left(\frac{1}{b}-\frac{M b^{\frac{p-1}{p+1}}}{(p+1) \epsilon^{p+1}}\right) v^{\frac{N}{N-2}} \leq 0 .
$$

Notice that

$$
\frac{1}{b}-\frac{M b^{\frac{p-1}{p+1}}}{(p+1) \epsilon^{p+1}}=0 \Longleftrightarrow \epsilon=\left(\frac{M}{p+1}\right)^{\frac{1}{p+1}} b^{\frac{2 p}{(p+1)^{2}}},
$$

and therefore

$$
1-b-\frac{M p b^{\frac{p-1}{p+1}} \epsilon^{\frac{p+1}{p}}}{p+1}=1-b-p b\left(\frac{M}{p+1}\right)^{\frac{p+1}{p}} .
$$

This coefficient vanishes if

$$
p\left(\frac{M}{p+1}\right)^{\frac{p+1}{p}}=\frac{p(N-1)-(N+1)}{2} .
$$

Therefore, if $M$ satisfies

$$
p\left(\frac{M}{p+1}\right)^{\frac{p+1}{p}}=\frac{p(N-2)-N}{2},
$$

we can choose $\epsilon>0$ so that the coefficient of $v^{(p-1) b+1}$ in (2.34) is equal to some $\tau>0$. Therefore $v$ satisfies

$$
-\Delta v+\tau v^{\frac{N}{N-2}} \leq 0 \quad \text { in } \Omega \backslash\{0\} .
$$

It follows by [13], $v \leq \max v$ and the same type of uniform estimate holds for $u$. This ends the case $p>\frac{N}{N-2}$.

Step 3. Finally we assume $p=\frac{N}{N-2}$ and $1<q<\frac{2 p}{p+1}=\frac{N}{N-1}$. From (2.12),

$$
M|\nabla u(x)|^{q} \leq c_{9}|x|^{-q \frac{p+1}{p-1}}=c_{9}|x|^{-q(N-1)}:=Q(x) .
$$

and $Q \in L^{1}\left(B_{2 R_{0}}\right)$. Let $\left\{\sigma_{n}\right\} \subset C_{0}^{\infty}\left(\mathbb{R}^{N}\right)$ such that $0 \leq \sigma_{n} \leq 1$

$$
\sigma_{n}(x)= \begin{cases}1 & \text { if } \frac{2}{n} \leq|x| \leq R_{0} \\ 0 & \text { if }|x| \in\left[0, \frac{1}{n}\right] \cup\left[2 R_{0}, \infty\right)\end{cases}
$$


and

$$
\left|\Delta \sigma_{n}\right| \leq 2 N n^{2} \chi_{B_{\frac{2}{n}} \backslash B_{\frac{1}{n}}}+\phi,
$$

where $\phi$ is a smooth nonnegative function with support in $B_{2 R_{0}} \backslash B_{R_{0}}$. Then

$$
-\int_{\left\{\frac{1}{n} \leq|x| \leq \frac{2}{n}\right\}} u \Delta \sigma_{n} d x-\int_{\left\{R_{0} \leq|x| \leq 2 R_{0}\right\}} u \Delta \sigma_{n} d x+\int_{\frac{1}{n} \leq|x|} u^{p} \sigma_{n} d x=M \int_{\frac{1}{n} \leq|x|}|\nabla u|^{q} \sigma_{n} d x .
$$

The right-hand side of (2.36) is bounded since $|\nabla u| \in L^{q}\left(B_{2 R_{0}}\right)$, the second term on the left is also uniformly bounded. Using the fact that $|x|^{N-2} u(x)$ is bounded by (2.1), we get

$$
\left|\int_{\left\{\frac{1}{n} \leq|x| \leq \frac{2}{n}\right\}} u \Delta \sigma_{n} d x\right| \leq C,
$$

for some $C>0$ independent of $n$. Letting $n \rightarrow \infty$ we infer that $u \in L_{l o c}^{p}(\Omega)$. By the maximum principle

$$
u(x) \leq u_{1}(x)=C \mathbf{G}^{B_{2 R_{0}}}[Q](x)+\max _{|z|=2 R_{0}} u(z),
$$

where $\mathbf{G}^{B_{2 R_{0}}}$ denotes the Green kernel in $B_{2 R_{0}}$. Since $Q(x)=C|x|^{-q(N-1)}$, a direct computation shows that $u_{1}(x) \leq c_{N} C|x|^{2-q(N-1)}=c_{N} C|x|^{2-N+\epsilon}$ for some $\epsilon>0$. We can write (1.1) under the form

$$
-\Delta u+c(x) u+d(x)|\nabla u|=0 \quad \text { in } \Omega \backslash\{0\},
$$

with $c(x)=u^{\frac{2}{N-2}}$ and $d(x)=|\nabla u|^{q-1}$. Then $c \in L^{\frac{N}{2}+\epsilon_{1}}\left(B_{2 R_{0}}\right)$ and $d \in$ $L^{N+\epsilon_{2}}\left(B_{2 R_{0}}\right)$; with $\epsilon_{1}, \epsilon_{2}>0$. It follows from [25, Theorem 10] that 0 is a removable singularity for $u$ in the sense that it can be extended as a $C^{2}$ solution in $\Omega$.

When the conditions of the theorem are not fulfilled there exist singular solutions. However these singular solutions may exhibit different types of behaviour according $1<q<\frac{2 p}{p+1}, \frac{2 p}{p+1}<q<2$ and $q=\frac{2 p}{p+1}$. In this case there may exist radial separable solutions of (1.9) under the form $u_{X}(r)=X r^{-\frac{2}{p-1}}$. Setting $\alpha=\frac{2}{p-1}$, then $X$ satisfies

$$
\Phi_{p}(X):=X^{p-1}-M \alpha^{\frac{2 p}{p+1}} X^{\frac{p-1}{p+1}}+\alpha(N-2-\alpha)=0
$$

The following result is easy to prove by a standard analysis of the function $\Phi_{p}$.

Proposition 2.9 Let $p>1$ and $M \in \mathbb{R}$.

(i) If $M$ is arbitrary and $1<p<\frac{N}{N-2}$, or $M>0$ and $p=\frac{N}{N-2}$, there exists one and only one positive solution $X_{1}$ to (2.38).

(ii) If $p>\frac{N}{N-2}$ and $M>m^{*}$, there exist two positive solutions $X_{1}<X_{2}$ to (2.38).

(iii) If $p>\frac{N}{N-2}$ and $M=m^{*}$ there exists one positive solution $X_{1}$ to (2.38).

(iv) If $p>\frac{N}{N-2}$ and $0<M<m^{*}$, or $M \leq 0$ and $p \geq \frac{N}{N-2}$, there exists no positive solution to (2.38). 
When $q \neq \frac{2 p}{p+1}$ the existence of singular solutions is much involved. It is developed in the subsequent paper [8].

Remark. It is noticeable that in the case $q=\frac{2 p}{p+1}, p>\frac{N}{N-2}$ and $M \geq m^{*}$, the equation exhibits a phenomenon which is characteristic of Lane-Emden type equations

$$
-\Delta u=u^{p} \quad \text { in } B_{1} \backslash\{0\} .
$$

If $u$ is nonnegative then there exists $\alpha \geq 0$ such that

$$
-\Delta u=u^{p}+\alpha \delta_{0} \quad \text { in } \mathcal{D}^{\prime}\left(B_{1}\right) .
$$

If $1<p<\frac{N}{N-2}$ then $\alpha$ can be positive, but if $p \geq \frac{N}{N-2}$, then $\alpha=0$. This means that the singularity cannot be seen in the sense of distributions, however there truly exist singular solutions, e.g. if $p>\frac{N}{N-2}$,

$$
u_{s}(x)=c_{N, p}|x|^{-\frac{2}{p-1}} \text {. }
$$

Here also for $q=\frac{2 p}{p+1}, p>\frac{N}{N-2}$, the isolated singularities are not seen in the sense of distributions.

\subsection{Proof of Theorem 1.2}

As in the proof of Theorem 1.1 we distinguish according $1<q<\frac{2 p}{p+1}$ or $q=\frac{2 p}{p+1}$. Without loss of generality we can suppose that $u>0$. We perform the same change of unknown as in the previous theorem putting $u=v^{b}$, but now we choose $b$ as follows

$$
(p-1) b+1=r \Longleftrightarrow b=\frac{r-1}{p-1},
$$

and we first assume that

$$
1-b-M \frac{q b^{q-1} \epsilon^{\frac{2}{q}}}{2}=0 \Longleftrightarrow \epsilon=\left(\frac{2(1-b)}{M q b^{q-1}}\right)^{\frac{q}{2}}=\left(\frac{2(p-r)}{M q(p-1) b^{q-1}}\right)^{\frac{q}{2}} .
$$

Hence (2.28) becomes

$$
-\Delta v+\frac{p-1}{r-1} v^{r}-\frac{(2-q) b^{q-1}}{2}\left(\frac{q}{2(1-b)}\right)^{\frac{q}{2-q}} M^{\frac{2}{2-q}} v^{\frac{(2 r-p-1) q+2(p-r)}{(p-1)(2-q)}} \leq 0 .
$$

Condition $r \geq \frac{(2 r-p-1) q+2(p-r)}{(p-1)(2-q)}$ is equivalent to $2 p-q(p+1) \leq r(2 p-q(p+1))$, since $1<r<p$.

Assuming first that $q<\frac{2 p}{p+1}$, we obtain from (2.44)

$$
-\Delta v+\frac{p-1}{2(r-1)} v^{r} \leq A .
$$

for some constant $A \geq 0$. Since $c a p_{2, r^{\prime}}^{\mathbb{R}^{N}}(K)=0$ the function $v$ is bounded from [3] and $v \leq c A^{\frac{1}{r}}+\max _{\partial \Omega} v$ for some $c>0$, hence $u$ is also uniformly upper bounded in $\Omega$ by some constant $a$. 
Next we have to show that $\nabla u \in L^{q}(\Omega)$. Let $\left\{\rho_{n}\right\}$ be a sequence of $C_{0}^{\infty}(\Omega)$ nonnegative functions such that $0 \leq \rho_{n} \leq 1, \rho_{n}=1$ in a small enough neighborhood of $K$ and $\left\|\rho_{n}\right\|_{W^{2, r^{\prime}}} \rightarrow 0$ when $n \rightarrow \infty$, and set $\eta_{n}=1-\rho_{n}$. Since

$$
\int_{\Omega} u \Delta \rho_{n} d x-\int_{\partial \Omega} \frac{\partial u}{\partial \mathbf{n}} d S+\int_{\Omega} u^{p} \eta_{n} d x=M \int_{\Omega}|\nabla u|^{q} \eta_{n} d x
$$

and

$$
\left|\int_{\Omega} u \Delta \rho_{n} d x\right| \leq c\|u\|_{L^{\infty}}\left\|\rho_{n}\right\|_{W^{2, r^{\prime}}} \rightarrow 0 \quad \text { as } n \rightarrow \infty
$$

we get

$$
\int_{\Omega} u^{p} d x-\int_{\partial \Omega} \frac{\partial u}{\partial \mathbf{n}} d S=M \int_{\Omega}|\nabla u|^{q} d x
$$

Hence $\nabla u \in L^{q}(\Omega)$. If $\zeta \in C_{0}^{\infty}(\Omega)$ and $\zeta_{n}=\zeta \eta_{n}$, there holds

$$
-\int_{\Omega} \eta_{n} u \Delta \zeta d x+\int_{\Omega} \zeta u \Delta \rho_{n} d x+\int_{\Omega} u^{p} \zeta_{n} d x=M \int_{\Omega}|\nabla u|^{q} \zeta_{n} d x .
$$

Since the second term on the left-hand side tends to 0 and $\zeta_{n} \rightarrow \zeta$ when $n \rightarrow \infty$, we obtain that

$$
-\int_{\Omega} u \Delta \zeta d x+\int_{\Omega} u^{p} \zeta d x=M \int_{\Omega}|\nabla u|^{q} \zeta d x
$$

Hence $u$ is a solution in the sense of distribution in $\Omega$.

Next we show that $\nabla u \in L^{2}(\Omega)$. Multiplying (1.9) by $u \eta_{n}$ and integrating, we obtain

$$
\int_{\Omega}|\nabla u|^{2} \eta_{n} d x-\int_{\Omega} u\left\langle\nabla u, \nabla \rho_{n}\right\rangle d x-\int_{\partial \Omega} u \frac{\partial u}{\partial \mathbf{n}} d S+\int_{\Omega} u^{p+1} \eta_{n} d x=M \int_{\Omega} u|\nabla u|^{q} \eta_{n} d x
$$

As

$$
\begin{aligned}
\int_{\Omega} u\left\langle\nabla u, \nabla \rho_{n}\right\rangle d x & =\frac{1}{2} \int_{\Omega}\left\langle\nabla u^{2}, \nabla \rho_{n}\right\rangle d x \\
& =-\frac{1}{2} \int_{\Omega} u^{2} \Delta \rho_{n} d x
\end{aligned}
$$

and

$$
\left|\int_{\Omega} u^{2} \Delta \rho_{n} d x\right| \leq c\|u\|_{L^{\infty}}^{2}\left\|\rho_{n}\right\|_{W^{2, r^{\prime}}}=o(1) \quad \text { as } n \rightarrow \infty,
$$

we infer that

$$
\int_{\Omega}|\nabla u|^{2} d x-\int_{\partial \Omega} u \frac{\partial u}{\partial \mathbf{n}} d S+\int_{\Omega} u^{p+1} d x=M \int_{\Omega} u|\nabla u|^{q} d x .
$$

Finally if $\zeta \in C_{0}^{\infty}(\Omega)$ and $\zeta_{n}=\zeta \eta_{n}$, then

$$
\int_{\Omega} \eta_{n}\langle\nabla u, \nabla \zeta\rangle d x-\int_{\Omega} \zeta\left\langle\nabla u, \nabla \rho_{n}\right\rangle d x+\int_{\Omega} u^{p} \zeta_{n} d x=M \int_{\Omega}|\nabla u|^{q} \zeta_{n} d x .
$$

Since $r \leq \frac{2 N}{N-2}$ there holds

$$
\left\|\rho_{n}\right\|_{W^{1,2}} \leq c\left\|\rho_{n}\right\|_{W^{2, r^{\prime}}} \Longrightarrow\left\|\rho_{n}\right\|_{W^{1,2}} \rightarrow 0 \quad \text { as } n \rightarrow \infty .
$$


Using the fact that $\nabla u \in L^{2}(\Omega)$ and Hölder's inequality, we derive

$$
\int_{\Omega} \zeta\left\langle\nabla u, \nabla \rho_{n}\right\rangle d x \rightarrow 0 \quad \text { as } n \rightarrow \infty .
$$

Hence

$$
\int_{\Omega}\langle\nabla u, \nabla \zeta\rangle d x+\int_{\Omega} u^{p} \zeta d x=M \int_{\Omega}|\nabla u|^{q} \zeta d x .
$$

This implies that $u$ is a weak solution of (1.9) and it is therefore $C^{2}$ in $\Omega$.

Next we assume $q=\frac{2 p}{p+1}$. We choose $b=\frac{r-1}{p-1}$ and (2.31) becomes

$$
-\Delta v+\left(1-b-\frac{M p b^{\frac{p-1}{p+1}} \epsilon^{\frac{p+1}{p}}}{p+1}\right) \frac{|\nabla v|^{2}}{v}+\left(\frac{1}{b}-\frac{M b^{\frac{p-1}{p+1}}}{(p+1) \epsilon^{p+1}}\right) v^{r} \leq 0 .
$$

If (2.32) holds with this choice of $b, 2.33$ ) becomes

$$
\begin{aligned}
1-b-\frac{M p b^{\frac{p-1}{p+1}} \epsilon^{\frac{p+1}{p}}}{p+1} & =1-b-p b\left(\frac{M}{p+1}\right)^{\frac{p+1}{p}} \\
& =\frac{1}{p-1}\left(p-r-p(r-1)\left(\frac{M}{p+1}\right)^{\frac{p+1}{p}}\right) .
\end{aligned}
$$

If $M<m_{r}^{*}$ defined by (1.10), we can choose $\epsilon$ such that

$$
1-b-\frac{M p b^{\frac{p-1}{p+1}} \epsilon^{\frac{p+1}{p}}}{p+1}=0
$$

and

$$
\frac{1}{b}-\frac{M b^{\frac{p-1}{p+1}}}{(p+1) \epsilon^{p+1}}=\tau:=\tau(\epsilon)>0
$$

Then $v$ satisfies

$$
-\Delta v+\tau v^{r} \leq 0 \quad \text { in } \Omega \backslash K .
$$

Since $\operatorname{cap}_{2, r^{\prime}}^{\mathbb{R}^{N}}(K)=0$ it follows from [3] that $v \leq \max _{x \in \partial \Omega} v(x)$. Hence $u$ is bounded. The different steps of the proof in the first case applies without any modification: first $\nabla u \in L^{q}(\Omega)$ and the equation holds in the sense of distributions in $\Omega$, then $\nabla u \in L^{2}(\Omega)$ and since $r \leq \frac{2 N}{N-2}$ we infer that $u$ is a weak solution and thus a strong one.

\section{Measure data}

Let $\Omega \subset \mathbb{R}^{N}$ be a bounded smooth domain with diameter smaller than $2 R$. Also any Radon measure in $\Omega$ is extended by 0 in $\Omega^{c}$ with the same notation.

\subsection{Proof of Theorem 1.3: the case $1<q<\frac{N}{N-1}$}

If $1<q<\frac{N}{N-1}$ assumption (1.12) with $\mu \geq 0$ reduces to

$$
\mu(K) \leq C \operatorname{cap}_{2, p^{\prime}}^{\mathbb{R}^{N}}(K) \quad \text { for all compact set } K \subset \Omega \text {. }
$$


The construction of solutions is based upon the following result due to BoccardoMurat-Puel [10]. It is concerned with a general quasilinear equation in a domain $G \subset \mathbb{R}^{N}$

$$
\mathcal{Q}(u):=-\Delta u+B(., u, \nabla u)=0 \quad \text { in } \mathcal{D}^{\prime}(G),
$$

where $B \in C\left(G \times \mathbb{R} \times \mathbb{R}^{N}\right)$ satisfies

$$
|B(x, r, \xi)| \leq \Gamma(|r|)\left(1+|\xi|^{2}\right) \text { for all }(x, r, \xi) \in G \times \mathbb{R} \times \mathbb{R}^{N},
$$

for some continuous increasing function $\Gamma$ from $\mathbb{R}^{+}$to $\mathbb{R}^{+}$.

Theorem 3.1 Let $G$ be a bounded domain in $\mathbb{R}^{N}$. If there exists a supersolution $\phi$ and a subsolution $\psi$ of the equation $\mathcal{Q} v=0$ belonging to $W^{1, \infty}(G)$ and such that $\psi \leq \phi$, then for any $\chi \in W^{1, \infty}(G)$ satisfying $\psi \leq \chi \leq \phi$ there exists a function $u \in W^{1,2}(G)$ solution of $\mathcal{Q} u=0$ such that $\psi \leq u \leq \phi$ and $u-\chi \in W_{0}^{1,2}(G)$.

The sub and super solutions are linked to the two problems in which $p$ and $q$ are bigger than 1 , and $\mu$ and $\omega$ are Radon measures

$$
\begin{aligned}
-\Delta v+|v|^{p-1} v & =\mu & & \text { in } \Omega \\
v & =0 & & \text { in } \partial \Omega,
\end{aligned}
$$

and

$$
\begin{aligned}
-\Delta w-M|\nabla w|^{q} & =\omega & & \text { in } \Omega \\
w & =0 & & \text { in } \partial \Omega .
\end{aligned}
$$

It is proved in [3, Theorem 4.1] that Problem (3.4) admits a solution, $v \in L^{p}(\Omega)$, necessarily unique, if and only if $\mu$ is absolutely continuous with respect to the Bessel capacity $c a p_{2, p^{\prime}}^{\mathbb{R}^{N}}$, that is

$$
\text { For any compact set } E \subset \Omega, \operatorname{cap}_{2, p^{\prime}}^{\mathbb{R}^{N}}(E)=0 \Longrightarrow|\mu|(E)=0 \text {. }
$$

Concerning (3.5), from [21, Theorem 1.9] a sufficient condition for solvability is the estimate

$$
\text { For any compact set } E \subset \Omega,|\omega|(E) \leq C \operatorname{cap} \mathbb{R}_{1, q^{\prime}}^{\mathbb{R}^{N}}(E),
$$

for some $C>0$. When $\mu$ is nonnegative and has compact support in $\Omega$, this condition turns out to be necessary. If (3.7) is satisfied there exists $\epsilon_{0}>0$ such that (3.5) admits a solution with $\omega$ replaced by $\epsilon \omega$ with $0<\epsilon \leq \epsilon_{0}$. Furthermore $\nabla w \in L^{q}(\Omega)$ and the following estimates hold [9, Theorem 1.2],

$$
|\nabla w(x)| \leq c_{13} \epsilon \mathbf{I}_{1}^{2 R}[|\omega|](x),
$$

at least if $\omega$ has compact support or is a smooth function, and, with no such conditions on $\mu$,

$$
|w(x)| \leq c_{14} \epsilon \mathbf{G}^{\Omega}[|\omega|](x),
$$

with $c_{13}, c_{14}$ depending on $N$ and $q$, where $\mathbf{I}_{1}^{2 R}$ is the truncated Riesz potential in $\mathbb{R}^{N}$ defined for any measure $\mu$ by

$$
\mathbf{I}_{1}^{2 R}[\mu](x)=\int_{0}^{2 R} \frac{\mu\left(B_{\rho}(x)\right)}{\rho^{N-1}} \frac{d \rho}{\rho} \text { for all } x \in \mathbb{R}^{N},
$$

and $\mathbf{G}^{\Omega}$ the Green potential in $\Omega$. If $R=\infty$ we denote by $\mathbf{I}_{1}:=\mathbf{I}_{1}^{\infty}$ the classical Riesz potential and if $\Omega=\mathbb{R}^{N}$ the role of $\mathbf{G}^{\Omega}$ is played by the Newtownian potential $\mathbf{I}_{2}$. We start with the following easy result: 
Lemma 3.2 Let $r>1, k \in \mathbb{N}^{*}$ and $\mu \in \mathfrak{M}_{+}(\Omega)$. If $\mu \in W^{-k, r}(\Omega)$ is nonnegative, then there exists $C>0$ such that

$$
\mu(E) \leq C\left(\operatorname{cap}_{k, r^{\prime}}^{\Omega}(E)\right)^{\frac{1}{r^{\prime}}} \quad \text { for any compact set } E \subset \Omega .
$$

where $r^{\prime}=\frac{r}{r-1}$. Conversely when $k=1,2$ and $\mu$ satisfies

$$
\mu(E) \leq C \operatorname{cap}_{k, r^{\prime}}^{\Omega}(E) \quad \text { for all compact set } E \subset \Omega,
$$

for some $C>0$, then,

(i) if $k=2$ then $\mu \in W^{-2, r}(\Omega)$,

(ii) if $k=1$ then $\mu \in W^{-1, r}(\Omega)$.

Proof. Assume first that $\mu \in \mathfrak{M}_{+}(\Omega) \cap W^{-k, r}(\Omega)$. Let $\zeta \in C_{0}^{\infty}(\Omega)$ such that $0 \leq \zeta \leq 1$ and $\zeta \geq \chi_{K}$. Then

$$
\mu(E) \leq \int_{\Omega} \zeta d \mu=\langle\mu, \zeta\rangle \leq\|\zeta\|_{W_{0}^{k, r^{\prime}}}\|\mu\|_{W^{-k, r}} .
$$

By the definition of capacity

$$
\mu(K) \leq\|\mu\|_{W^{-k, r}}\left(\operatorname{cap}_{k, r p^{\prime}}^{\Omega}(K)\right)^{\frac{1}{r^{\prime}}} .
$$

Conversely if (3.12) holds with $k=2$ there exists $\epsilon_{0}>0$ such that for every $\epsilon \in\left(0, \epsilon_{0}\right]$, there exists $z \in L^{r}(\Omega)$ satisfying

$$
\begin{aligned}
-\Delta z & =z^{r}+\epsilon \mu & & \text { in } \Omega \\
z & =0 & & \text { on } \partial \Omega,
\end{aligned}
$$

(see [24, Theorem 2.10, Remark 2.11]). Since $z \geq \mathbf{G}^{\Omega}[\epsilon \mu]$, it follows that $\mathbf{G}^{\Omega}[\mu] \in L^{r}(\Omega)$ and therefore $\mu \in W^{-2, r}(\Omega)$. Since $\mathbf{G}^{\Omega}$ is an isomorphism from $L^{r^{\prime}}(\Omega)$ into $W^{2, r^{\prime}}(\Omega) \cap W_{0}^{1, r^{\prime}}(\Omega)$, we infer by duality that $\mathbf{G}^{\Omega}$ is an isomorphism from $W^{-2, r}(\Omega)$ into $L^{r}(\Omega)$. Hence $\mu \in W^{-2, r}(\Omega)$.

Finally, if (3.12) holds with $k=1$, then there exists $\epsilon_{0}>0$ such that for every $\epsilon \in\left(0, \epsilon_{0}\right]$ there exists $z \in W^{1, r}(\Omega)$ satisfying

$$
\begin{aligned}
-\Delta z & =|\nabla z|^{r}+\epsilon \mu & & \text { in } \Omega \\
z & =0 & & \text { on } \partial \Omega .
\end{aligned}
$$

Then $z$ satisfies $z \geq \mathbf{G}^{\Omega}[\epsilon \mu]$. Since $z \in L^{r^{*}}(\Omega)$ by Sobolev imbedding theorem, we have that $\mathbf{G}^{\Omega}[\mu] \in L^{r^{*}}(\Omega)$, which implies the claim.

Proof of the theorem. We put $\mu_{n}=\mu * \eta_{n}$ where $\left\{\eta_{n}\right\} \subset C_{0}^{\infty}\left(\mathbb{R}^{N}\right)$ is a sequence of mollifiers with $\operatorname{supp}\left(\eta_{n}\right) \subset B_{\frac{1}{n}}$, and we denote by $v_{n}$ the solution of

$$
\begin{aligned}
-\Delta v+v^{p} & =\epsilon \mu_{n} \chi_{\Omega} & & \text { in } \Omega \\
v & =0 & & \text { on } \partial \Omega .
\end{aligned}
$$

Since $\mu$ satisfies (3.12) so does $\mu_{n}$ with the same constant $C$. Hence $\mu_{n} \in$ $W^{-2, p}(\Omega)$ and $\mu_{n} \rightarrow \mu$ in $W^{-2, p}(\Omega)$ as $n \rightarrow \infty$. We also denote by $z_{n}$ a nonnegative solution of

$$
\begin{aligned}
-\Delta z & =z^{p}+\epsilon \mu_{n} \chi_{\Omega} & & \text { in } \Omega \\
z & =0 & & \text { on } \partial \Omega,
\end{aligned}
$$


and by $w_{n}$ a nonnegative solution of

$$
\begin{aligned}
-\Delta w & =M|\nabla w|^{q}+\epsilon \mu_{n} \chi_{\Omega} & & \text { in } \Omega \\
w & =0 & & \text { on } \partial \Omega,
\end{aligned}
$$

Since $w_{n}$ is $C^{2}$, it is unique by the strong maximum principle. Then there holds by (3.8), (3.9),

$$
\begin{aligned}
& v_{n} \leq \epsilon \mathbf{G}^{\Omega}\left[\mu_{n}\right] \leq w_{n} \leq c_{14} \epsilon \mathbf{G}^{\Omega}\left[\mu_{n}\right] \leq\left. c_{14} \epsilon \mathbf{I}_{2}\left[\mu_{n}\right]\right|_{\Omega}, \\
& \left|\nabla w_{n}\right| \leq c_{13} \epsilon \mathbf{I}_{1}^{2 R}\left[\mu_{n}\right] .
\end{aligned}
$$

Since $v_{n}$ and $w_{n}$ are respectively a subsolution and a supersolution of

$$
\begin{aligned}
-\Delta u+u^{p} & =M|\nabla u|^{q}+\epsilon \mu_{n} & & \text { in } \Omega \\
u & =0 & & \text { on } \partial \Omega,
\end{aligned}
$$

it follows by Theorem 3.1 that there exists $u=u_{n} \in W_{0}^{1, \infty}(\Omega)$ satisfying (3.19) in the sense that for any $\zeta \in C_{c}^{2}(\bar{\Omega})$ there holds

$$
-\int_{\Omega} u_{n} \Delta \zeta d x+\int_{\Omega}\left(u_{n}^{p}-M\left|\nabla u_{n}\right|^{q}\right) \zeta d x=\epsilon \int_{\Omega} \zeta d \mu_{n} .
$$

It is unique by the strong maximum principle and it satisfies

$$
v_{n} \leq u_{n} \leq w_{n} \leq c_{14} \epsilon \mathbf{I}_{2}\left[\mu_{n}\right]\left\lfloor_{\Omega} .\right.
$$

Since $\mathbf{I}_{2}\left[\mu_{n}\right]\left\lfloor\Omega\right.$ is uniformly bounded in $L^{p}(\Omega)$, the sequence of functions $\left\{u_{n}\right\}$ shares this property. If $\eta=\mathbf{G}^{\Omega}[1]$, there holds

$$
\int_{\Omega}\left(u_{n}+\eta u_{n}^{p}\right) d x=M \int_{\Omega}\left|\nabla u_{n}\right|{ }^{q} \eta d x+\epsilon \int_{\Omega} \eta d \mu_{n} .
$$

Hence $\left|\nabla u_{n}\right|$ is uniformly bounded in $L_{d_{\Omega}}^{q}(\Omega)$ where $d_{\Omega}(x)=\operatorname{dist}(x, \partial \Omega)$. By (3.8),

$$
\begin{aligned}
\left|\nabla u_{n}\right| \leq c_{13} \epsilon \mathbf{I}_{1}^{2 R}\left[\left|\mu_{n}-u_{n}^{p}\right|\right] & \leq c_{13}\left(\epsilon \mathbf{I}_{1}^{2 R}\left[\mu_{n}\right]+\mathbf{I}_{1}^{2 R}\left[u_{n}^{p}\right]\right) \\
& \leq c_{13}\left(\epsilon \mathbf{I}_{1}^{2 R}\left[\mu_{n}\right]+c_{14}^{p} \epsilon^{p} \mathbf{I}_{1}^{2 R}\left[\left(\mathbf{I}_{2}\left[\mu_{n}\lfloor\Omega]\right)^{p}\right]\right) .\right.
\end{aligned}
$$

Using [16, Lemma 4.2] we have equivalence between

$$
\mathbf{I}_{1}\left[\left(\mathbf{I}_{2}\left[\mu_{n}\lfloor\Omega]\right)^{p}\right] \leq c_{15} \mathbf{I}_{1}\left[\mu_{n}\lfloor\Omega]\right.\right.
$$

and

$$
\mathbf{I}_{2}\left[\left(\mathbf{I}_{2}\left[\mu_{n}\lfloor\Omega]\right)^{p}\right] \leq c_{17} \mathbf{I}_{2}\left[\mu_{n}\lfloor\Omega],\right.\right.
$$

and $c_{17} \leq c_{15} \leq C(N, p) c_{17}$. Moreover, since $\operatorname{diam}(\Omega)<2 R$,

$$
\left(\mathbf{I}_{1}^{2 R}\left[\left(\mathbf{I}_{2}[\mu]\right)^{p}\right]\right)^{q} \leq c_{21}^{q}\left(\mathbf{I}_{1}[\mu]\right)^{q} \leq c^{q} c_{21}^{q}\left(\mathbf{I}_{1}^{2 R}[\mu]\right)^{q} .
$$

for some $c=c(N, R)>0$. Next, it is quoted in [16. Theorem 1.1] that the inequality (3.25) is equivalent to the main assumption of Theorem 1.3 .

$$
\mu_{n}\left\lfloor_{\Omega}(E) \leq \operatorname{Ccap}_{2, p^{\prime}}^{\mathbb{R}^{N}}(E) \quad \text { for all compact set } E \subset \Omega,\right.
$$


for some $C>0$. Actually this equivalence is proved in [19]. By [1, Theorem 3.14-(a)] there exists $A=A(N)>0$ such that

$$
\mid\left\{x \in \mathbb{R}^{N}:\left|\mathbf{I}_{1}\left[\mu_{n}\lfloor\Omega](x) \mid>\lambda\right\}\right| \leq A \lambda^{-\frac{N}{N-1}} \| \mu_{n}\left\lfloor\Omega \|_{L^{1}}^{\frac{N}{N-1}} .\right.\right.
$$

Clearly the above inequality holds if $\mathbf{I}_{1}$ is replaced by $\mathbf{I}_{1}^{2 R}$ and $\mathbb{R}^{N}$ by $\Omega$. This is an estimate of $\mathbf{I}_{1}^{2 R}\left[\mu_{n}\lfloor\Omega]\right.$ in the Lorentz space $L^{\frac{N}{N-1}, \infty}(\Omega)$ (or Marcinkiewicz space). Clearly

$$
\| \mu_{n}\left\lfloor\Omega\left\|_{L^{1}} \leq\right\| \mu \|_{\mathfrak{M}} .\right.
$$

Therefore 3.23) implies that $\left|\nabla u_{n}\right|$ is bounded in $L^{\frac{N}{N-1}, \infty}(\Omega)$, hence equiintegrable in $L^{q}(\Omega)$ since $q<\frac{N}{N-1}$. By Lemma 3.2 (ii) and classical harmonic analysis results, $\mathbf{I}_{2}\left[\mu_{n} \iota_{\Omega} \rightarrow \mathbf{I}_{2}\left[\mu\left\lfloor_{\Omega}\right.\right.\right.$ in $L^{p}(\Omega)$ (see e.g. [26]). It follows from (3.21) that $u_{n}$ is equi-integrable in $L^{p}(\Omega)$. By standard results on elliptic equations and measure theory [11, Corollary IV], the sequences $\left\{u_{n}\right\}$ and $\left\{\left|\nabla u_{n}\right|\right\}$ are relatively compact in $L^{1}(\Omega)$. Hence there exist a subsequence $\left\{n_{j}\right\}$, converging to $\infty$ and a function $u \in W_{0}^{1, q}(\Omega) \cap L^{p}(\Omega)$ such that $u_{n_{j}} \rightarrow u$ in $W^{1,1}(\Omega)$ and a.e. in $\Omega$. Since $\left\{u_{n}\right\}$ and $\left\{\left|\nabla u_{n}\right|\right\}$ are also equi-integrable in $L^{p}(\Omega)$ and $L^{q}(\Omega)$ respectively, we infer that $u_{n_{j}} \rightarrow u$ in $W_{0}^{1, q}(\Omega) \cap L^{p}(\Omega)$. It follows from Vitali's convergence theorem that $u_{n_{j}} \rightarrow u$ in $L^{p}(\Omega)$ and $\nabla u_{n_{j}} \rightarrow \nabla u$ in $L^{q}(\Omega)$. Letting $n_{j} \rightarrow \infty$ in (3.20) we conclude that the identity

$$
-\int_{\Omega} u \Delta \zeta d x+\int_{\Omega}\left(u^{p}-M|\nabla u|^{q}\right) \zeta d x=\epsilon \int_{\Omega} \zeta d \mu_{n}
$$

holds for any $\zeta \in C_{c}^{2}(\bar{\Omega})$. Clearly

$$
v \leq u \leq w \leq C \epsilon \mathbf{I}_{2}[\mu]\left\lfloor_{\Omega},\right.
$$

where $v$ and $w$ are respectively the solution of (3.4) and the minimal solution of (3.5) with $\mu$ replaced by $\epsilon \mu$.

\subsection{Proof of Theorem 1.3; the general case}

The approach with super and sub solutions does not work directly and we follow the method developed for proving [21, Theorem 1.9] which is a delicate extension of the one in the subcritical case. The fact that a sequence of approximation $\left\{u_{n}\right\}$ is bounded in $W_{0}^{1, q}(\Omega) \cap L^{p}(\Omega)$ does not imply the uniform integrability of $\left\{\nabla u_{n}\right\}$ in $L^{q}(\Omega)$ for $q \geq \frac{N}{N-1}$.

Definition 3.3 If $r>1$ and $k \in \mathbb{N}^{*}$ we denote by $\mathbf{M}^{k, r}(\Omega)$ the set of bounded measures $\mu$ in $\Omega$ which satisfy, for some $C>0$,

$$
|\mu|(E) \mid \leq C_{c a p}^{\Omega}(E) \quad \text { for all compact sets } E \subset \Omega \text {. }
$$

If $\Omega$ is replaced by $\mathbb{R}^{N}$, the set is denoted by $\mathbf{M}^{k, r}$. The smallest constant $C$ such that (3.31) holds is denoted by $[\mu]_{M^{k, r}}$.

For $T>1$ we denote by $E_{1}(T, \mu)$ the subset of functions $\zeta \in W_{0}^{1, q}(\Omega)$ such that

$$
\int_{\Omega}|\nabla \zeta|^{q} w d x \leq T \epsilon^{q} \int_{\Omega}\left(\mathbf{I}_{1}^{2 R}[|\mu|]\right)^{q} w d x \quad \text { for all } w \in \mathbf{A}_{1} \cap L^{\infty} .
$$

The following estimate is obtained in [21, Lemma 5.2] in a more general context. 
Lemma 3.4 Let $q>1$ and $\mu \in \mathbf{M}^{1, q^{\prime}}(\Omega)$. Then there exists $c_{17}=c_{17}(N, q)>$ 0 such that for any $\zeta \in E_{1}(T, \mu)$

$$
\mathbf{I}_{1}^{2 R}\left[|\nabla \zeta|^{q} \chi_{\Omega}\right](x) \leq c_{17} T \epsilon^{q}[\mu]_{M^{1, q^{\prime}}}^{q-1} \mathbf{I}_{1}^{2 R}[|\mu|](x)
$$

a.e. in $\Omega$.

Lemma 3.5 Let $q>1, \zeta \in E_{1}(T, \mu)$ where $\mu \in \mathbf{M}^{1, q^{\prime}}(\Omega)$, and $S_{1}(\zeta)$ be the solution of

$$
\begin{aligned}
-\Delta \phi+\phi^{p} & =M|\nabla \zeta|^{q}+\epsilon \mu & & \text { in } \Omega \\
\phi & =0 & & \text { on } \partial \Omega .
\end{aligned}
$$

Then there exists $c_{18}=c_{18}(N, q)>0$ such that

$$
v \leq S_{1}(\zeta) \leq S(\zeta) \leq\left(\epsilon+c_{18} \epsilon^{q} T\right) I_{2}[\mu],
$$

where $S(\zeta)$ is the solution of

$$
\begin{aligned}
-\Delta \phi & =M|\nabla v|^{q}+\epsilon \mu & & \text { in } \Omega \\
\phi & =0 & & \text { on } \partial \Omega,
\end{aligned}
$$

and $v$ is the solution of (3.4) with $\mu$ replaced by $\epsilon \mu$.

Proof. There holds

$$
S_{1}(\zeta)=\epsilon \mathbf{G}^{\Omega}[\mu]+\mathbf{G}^{\Omega}\left[|\nabla \zeta|^{q}\right] \leq \epsilon \mathbf{I}_{2}[\mu]+\mathbf{I}_{2}\left[|\nabla \zeta|^{q}\right]=\epsilon \mathbf{I}_{2}[\mu]+\mathbf{I}_{1}\left[\mathbf{I}_{1}\left[|\nabla \zeta|^{q}\right]\right] .
$$

By Lemma 3.4 with $R=\infty, \mathbf{I}_{1}\left[|\nabla \zeta|^{q} \chi_{\Omega}\right] \leq c_{17} T \epsilon^{q}[\mu]_{M^{1, q^{q}}}^{q-1} \mathbf{I}_{1}[\mu]$, hence

$$
S_{1}(\zeta) \leq\left(\epsilon+c_{17} T \epsilon^{q}[\mu]_{M^{1, q^{\prime}}}^{q-1}\right) \mathbf{I}_{2}[\mu]:=\left(\epsilon+c_{18} \epsilon^{q} T\right) \mathbf{I}_{2}[\mu],
$$

since $T \geq 1$ and $\epsilon \in(0,1]$.

Lemma 3.6 There exists $T_{1}>0$ such that for any $T>T_{1}$, there exists $\epsilon_{T}>0$ such that for all $\epsilon \in\left(0, \epsilon_{T}\right], S_{1}$ maps $E(T, \mu)$ into itself.

Proof. By [21, Theorem 1.4], and using the proof of [21, Theorem 1.9], there exist constants $c_{19}, c_{20}, c_{21}>0$ depending on $N, q, R$ and $m$ such that for any $w \in \mathbf{A}_{1} \cap L^{\infty}$ such that $[w]_{A_{1}} \leq m$

$$
\begin{aligned}
& \int_{\Omega}\left|\nabla S_{1}(\zeta)\right|^{q} w d x \leq \leq c_{19} \int_{\Omega}\left(\mathbf{I}_{1}^{2 R}\left[|\nabla \zeta|^{q} \chi_{\Omega}\right]+\epsilon \mathbf{I}_{1}^{2 R}[\mu]+\mathbf{I}_{1}^{2 R}\left[S_{1}^{p}(\zeta)\right]\right)^{q} w d x \\
& \leq c_{20} \int_{\Omega}\left(\left(\mathbf{I}_{1}^{2 R}\left[|\nabla \zeta|^{q} \chi_{\Omega}\right]\right)^{q}+\epsilon^{q}\left(\mathbf{I}_{1}^{2 R}[\mu]\right)^{q}\right. \\
&\left.+\left(\epsilon^{p q}+c_{21} \epsilon^{p q^{2}} T^{p q}\right)\left(\mathbf{I}_{1}^{2 R}\left[\left(\mathbf{I}_{2}[\mu]\right)^{p}\right]\right)^{q}\right) w d x
\end{aligned}
$$

Using again the equivalence between (3.24), (3.25), (3.26) and (3.27), inequality (3.38) is transformed in

$$
\int_{\Omega}\left|\nabla S_{1}(\zeta)\right|^{q} w d x \leq c_{22}\left(\epsilon^{q}+c_{24} q T^{q} \epsilon^{q^{2}}+c_{25}^{q}\left(\epsilon^{p q}+c_{20} \epsilon^{p q^{2}} T^{p q}\right)\right) \int_{\Omega}\left(\mathbf{I}_{1}^{2 R}[\mu]\right)^{q} w d x .
$$


Finally for any $T>c_{23}$ there exists $\epsilon_{0}$ such that for $0<\epsilon \leq \epsilon_{0}$, there exist positive constants $c_{24}, c_{25}$ such that

$$
c_{22}\left(\epsilon^{q}+c_{24} q T^{q} \epsilon^{q^{2}}+c_{25}^{q}\left(\epsilon^{p q}+c_{20} \epsilon^{p q^{2}} T^{p q}\right)\right) \leq T \epsilon^{q},
$$

which implies the claim.

Lemma 3.7 Under the assumptions of Lemma 3.6 with $T>c_{23}$ and $0<\epsilon \leq$ $\epsilon_{T}$, the mapping $S_{1}$ is compact from $E(T, \mu)$ into itself.

Proof. We prove first the continuity. Let $\left\{\zeta_{j}\right\} \subset E(T, \mu)$ such that $\zeta_{j} \rightarrow \zeta$ in $W_{0}^{1, q}(\Omega)$. Using monotonicity as in [12, Theorem 8] we obtain that for any $r \in\left[1, \frac{N}{N-1}\right)$, there exists $\alpha=\alpha(N, R, r)>0$ such that

$$
\alpha\left\|S_{1}\left(\zeta_{j}\right)-S_{1}\left(\zeta_{\ell}\right)\right\|_{W_{0}^{1, r}}+\left\|S_{1}^{p}\left(\zeta_{j}\right)-S_{1}^{p}\left(\zeta_{\ell}\right)\right\|_{L^{1}} \leq M\left\|\left|\nabla \zeta_{j}\right|^{q}-\left|\nabla \zeta_{\ell}\right|^{q}\right\|_{L^{1}} .
$$

Since $\left\{\nabla \zeta_{j}\right\}$ is a Cauchy sequence in $L^{q}(\Omega)$ it follows that $S_{1}\left(\zeta_{j}\right) \rightarrow S_{1}(\zeta)$ in $W_{0}^{1, r}(\Omega) \cap L^{p}(\Omega)$. Hence

$$
\begin{aligned}
-\Delta S_{1}(\zeta)+S_{1}^{p}(\zeta) & =M|\nabla \zeta|^{q}+\mu & & \text { in } \Omega \\
S_{1}(\zeta) & =0 & & \text { in } \partial \Omega .
\end{aligned}
$$

If we take $\nu:=\nu_{j}=M\left|\nabla \zeta_{j}\right|^{q}+\mu+S_{1}^{p}\left(\zeta_{j}\right)$. By (3.26)

$$
\mathbf{I}_{1}^{2 R}\left[S_{1}^{p}\left(\zeta_{j}\right)\right] \leq \mathbf{I}_{1}^{2 R}\left[\left(\mathbf{I}_{2}[\mu]\right)^{p}\right] \leq C_{6} \mathbf{I}_{1}^{2 R}[\mu] .
$$

Combined with (3.33) we infer that

$$
\mathbf{I}_{1}^{2 R}\left[M\left|\nabla \zeta_{j}\right|^{q}+S_{1}^{p}\left(\zeta_{j}\right)+\mu\right] \leq c_{28} \mathbf{I}_{1}^{2 R}[\mu] .
$$

Let $\mathbf{M}_{1}$ be the first order fractional maximal function defined by

$$
\mathbf{M}_{1}(\mu)(x):=\sup _{\rho>0} \frac{\omega\left(B_{\rho}(x)\right)}{\rho^{N-1}} \quad \text { for all } x \in \mathbb{R}^{N} .
$$

It is classical that for all $\nu \in \mathfrak{M}_{b}(\Omega)$,

$$
\left.\mathbf{M}_{1}[|\nu|](x) \leq m_{N} \mathbf{I}_{1}^{2 R}[|\nu|]\right)(x) \quad \text { for a.e. } x \in \mathbb{R}^{N} .
$$

Hence, if we set $c_{29}=m_{N}\left(c_{28}+1\right)$, there holds.

$$
\mathbf{M}_{1}\left[M\left|\nabla \zeta_{j}\right|^{q}+\mu+S_{1}^{p}\left(\zeta_{j}\right)\right](x) \leq c_{29} \mathbf{I}_{1}^{2 R}[\mu] .
$$

Since $\mathbf{I}_{1}^{2 R}[\mu] \in L^{q}(\Omega)$ we deduce that the left-hand side of (3.45) is bounded and equi-integrable in $L^{q}(\Omega)$. Then we apply [21, Corollary 1.7], with $w=1$ and deduce that there exists a subsequence $\left\{\nabla S_{1}\left(\zeta_{j_{n}}\right)\right\}$ which converges in $L^{q}(\Omega)$. By uniqueness the limit is $\left\{\nabla S_{1}(\zeta)\right\}$ and the whole sequence $\left\{\nabla S_{1}\left(\zeta_{j}\right)\right\}$ converges. Therefore $S_{1}$ is continuous.

The proof of the compactness follows the same ideas: If $\left\{\zeta_{j}\right\}$ is a bounded sequence in $E(T, \mu)$, then $\left\{\nabla S_{1}\left(\zeta_{j}\right)\right\}$ is bounded in $L_{w}^{q}(\Omega)$ by (3.38), hence (3.40) holds. By (3.25), $\left\{S_{1}\left(\zeta_{j}\right)\right\}$ is bounded in $L^{p}(\Omega)$ and equi-integrable since $\mathbf{I}_{2}[\mu]$ belongs to $L^{p}(\Omega)$ (it is a consequence of (3.27)) and Lemma 3.2. By [11] the sequence $\left\{S_{1}\left(\zeta_{j}\right)\right\}$ is relatively compact in $W_{0}^{1,1}(\Omega)$. Hence, there exist 
$\zeta \in E(T, \mu)$ and a subsequence $\left\{\zeta_{j_{n}}\right\}$ such that $\zeta_{j_{n}} \rightarrow \zeta$ weakly in $W_{0}^{1, q}(\Omega)$ and $\left\{S_{1}\left(\zeta_{j_{n}}\right)\right\}$ converges to some $S \in E(T, \mu)$ in $W_{0}^{1,1}(\Omega) \cap L^{p}(\Omega)$, a.e. in $\Omega$ and weakly in $W_{0}^{1, q}(\Omega)$. As above we derive from [21, Corollary 1.7] that the sequence $\left\{\mathbf{M}_{1}\left[M\left|\nabla \zeta_{j_{n}}\right|^{q}+\mu+S_{1}^{p}\left(\zeta_{j}\right)\right]\right\}$ is bounded and equi-integrable in $L^{q}(\Omega)$, hence $\left\{\nabla S_{1}\left(\zeta_{j_{n}}\right)\right\}$ converges to $\nabla S$ in $L^{q}(\Omega)$. Therefore $S$ is a solution of

$$
\begin{aligned}
-\Delta S+S^{p} & =M|\nabla \zeta|^{q}+\mu & & \text { in } \Omega \\
S & =0 & & \text { on } \partial \Omega .
\end{aligned}
$$

This implies that $S=S_{1}(\zeta)$ and the mapping $S_{1}$ is compact.

End of the proof of Theorem 1.3 It follows from Lemma 3.7 that $S_{1}$ is a compact mapping from $E(T, \mu)$ into itself. Hence it admits a fixed point $u$ by Schauder's theorem and $u \in W_{0}^{1, q}(\Omega) \cap L^{p}(\Omega)$ is nonnegative and satisfies (1.13).

\subsection{Proof of the Corollaries}

Proof of Corollary 1.4. If $q$ satisfies $\frac{N p}{N+p} \leq q<2$, then $1<p<\frac{2 N}{N-2}$. By Sobolev imbedding theorem there holds

$$
\|\phi\|_{W^{1, q^{\prime}}} \leq c_{27}\|\phi\|_{W^{2, p^{\prime}}} \quad \text { for all } \phi \in C^{2}(\bar{\Omega}),
$$

where $c_{27}$ depends on $p, q$ and $|\Omega|$, provided

$$
\frac{1}{q^{\prime}} \geq \frac{1}{p^{\prime}}-\frac{1}{N} \Longleftrightarrow q \geq \frac{N p}{N+p} .
$$

The condition $q<2$ necessitates that $\frac{N p}{N+p}<2$, equivalently $p<\frac{2 N}{N+2}$.

Proof of Corollary 1.5. We recall [1, Theorem 5.5.1] (a)-(b). If $2 p^{\prime} \leq q^{\prime}<N$, then

$$
\left(\operatorname{cap}_{2, p^{\prime}}^{\mathbb{R}^{N}}(E)\right)^{\frac{1}{N-2 p^{\prime}}} \leq A\left(\operatorname{cap}_{1, q^{\prime}}^{\mathbb{R}^{N}}(E)\right)^{\frac{1}{N-q}} \quad \text { for all Borel set } E \subset \Omega .
$$

Since $\frac{N-2 p^{\prime}}{N-q^{\prime}} \geq 1$ we deduce

$$
\operatorname{cap} p_{2, p^{\prime}}^{\mathbb{R}^{N}}(E) \leq A^{\prime} \operatorname{cap}_{1, q^{\prime}}^{\mathbb{R}^{N}}(E) \quad \text { for all Borel set } E \subset \Omega .
$$

Hence (1.15) implies (1.12) and existence follows from Theorem 1.3. The assumption $2 p^{\prime} \leq q^{\prime}<N$ is equivalent to $\frac{N}{N-1}<q \leq \frac{2 p}{p+1}$. Note that this implies $p>\frac{N}{N-2}$.

Proof of Corollary 1.6. -(i) Since $p$ and $q$ are subcritical, (1.12) is verified as soon as there holds for every Borel set $E \subset \Omega$,

$$
\mu(E) \leq \frac{\mu(\Omega)}{\min \left\{c a p_{2, p^{\prime}}^{\mathbb{R}^{N}}(\{0\}), c a p_{1, q^{\prime}}^{\mathbb{R}^{N}}(\{0\})\right\}} \min \left\{c a p_{2, p^{\prime}}^{\mathbb{R}^{N}}(E), \operatorname{cap} p_{1, q^{\prime}}^{\mathbb{R}^{N}}(E)\right\} .
$$

-(ii) If $p$ is subcritical and $q$ supercritical, (1.14) implies (1.12).

-(iii) If $q$ is subcritical and $\mu$ satisfies (1.15) then (1.12) holds and the existence follows by Theorem 1.3 However the condition that $\mu$ vanishes on Borel sets 


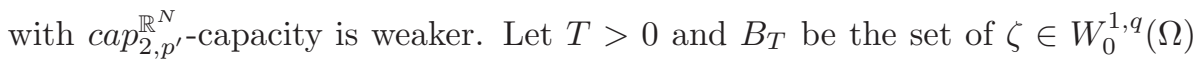
such that $\left\|M|\nabla \zeta|^{q}\right\|_{L^{1}(\Omega)} \leq T$. If $\zeta \in B_{T}$ there exists a unique solution $S(\zeta) \in$ $W_{0}^{1,1}(\Omega) \cap L^{p}(\Omega)$ to

$$
\begin{aligned}
-\Delta \phi+\phi^{p} & =M|\nabla \zeta|^{q}+\mu & & \text { in } \Omega \\
\phi & =0 & & \text { on } \partial \Omega .
\end{aligned}
$$

This follows from [3] since the right-hand side of the equation is a measure absolutely continuous with respect to the $c a p_{2, p^{\prime}}^{\mathbb{R}^{N}}$-capacity and the solution is nonnegative. The following estimate holds [12, Theorem 8]

$$
\begin{aligned}
& \int_{\Omega} S^{p}(\zeta) d x \leq \mu(\Omega)+M \int_{\Omega}|\nabla \zeta|^{q} d x \\
& \alpha\|S(\zeta)\|_{W_{0}^{1, r}} \leq 2 \mu(\Omega)+2 M \int_{\Omega}|\nabla \zeta|^{q} d x,
\end{aligned}
$$

for any $1<r<\frac{N}{N-1}$. This implies in the case $q=r$,

$$
\left(\int_{\Omega}|\nabla S(\zeta)|^{q} d x\right)^{\frac{1}{q}} \leq A \mu(\Omega)+B \int_{\Omega}|\nabla \zeta|^{q} d x
$$

with

$$
A=\frac{2}{\alpha} \text { and } B=\frac{2 M}{\alpha} .
$$

For $X>0$ set $F(X)=B X^{q}-X+A \mu(\Omega)$. Then $F^{\prime}(X)=0$ iff $X=X_{0}:=$ $(q B)^{-\frac{1}{q-1}}$ and

$$
F\left(X_{0}\right)=A \mu(\Omega)-\frac{q-1}{q}(q B)^{-\frac{1}{q-1}} .
$$

If we assume that

$$
F\left(X_{0}\right)<0 \Longleftrightarrow \mu(\Omega)<\frac{q-1}{q A}(q B)^{-\frac{1}{q-1}}=(q-1)\left(\frac{\alpha}{2}\right)^{\frac{q}{q-1}} M^{-\frac{1}{q-1}},
$$

then $\min F(X)<0$, therefore $F$ admits two positive roots $X_{2}<X_{0}<X_{1}$. Hence the inequality

$$
X_{2}<\left(\int_{\Omega}|\nabla \zeta|^{q} d x\right)^{\frac{1}{q}} \leq X_{1}
$$

implies

$$
\left(\int_{\Omega}|\nabla S(\zeta)|^{q} d x\right)^{\frac{1}{q}} \leq B \int_{\Omega}|\nabla \zeta|^{q} d x+A \mu(\Omega) \leq\left(\int_{\Omega}|\nabla \zeta|^{q} d x\right)^{\frac{1}{q}} \leq X_{1},
$$

and if

$$
\left(\int_{\Omega}|\nabla \zeta|^{q} d x\right)^{\frac{1}{q}} \leq X_{2}
$$

then

$$
\left(\int_{\Omega}|\nabla S(\zeta)|^{q} d x\right)^{\frac{1}{q}} \leq A \mu(\Omega)+B X_{2}^{q} \leq A \mu(\Omega)+B X_{1}^{q}=X_{1} .
$$


Therefore the mapping $S$ sends $B_{X_{1}}$ into itself. It is continuous since

$$
\begin{aligned}
& \int_{\Omega}\left|S^{p}\left(\zeta_{1}\right)-S^{p}\left(\zeta_{2}\right)\right| \leq M \int_{\Omega} \|\left.\nabla \zeta_{1}\right|^{q}-\left|\nabla \zeta_{2}\right|^{q} \mid d x \\
& \alpha\left\|S\left(\zeta_{1}\right)-S\left(\zeta_{2}\right)\right\|_{W_{0}^{1, r}} \leq 2 M \int_{\Omega} \|\left.\nabla \zeta_{1}\right|^{q}-\left|\nabla \zeta_{2}\right|^{q} \mid d x .
\end{aligned}
$$

The fact that this operator is compact follows from the a priori estimate (3.50) and Lemma 3.7. The conclusion is a consequence of Schauder Theorem.

\section{References}

[1] D. Adams and L. Hedberg. Function Spaces and Potential Theory, Theory, Springer-Verlag, London-Berlin-Heidelberg-New York (1996).

[2] S. Alarcon, J. García-Melián and A. Quass. Keller-Osserman type conditions for some elliptic problems with gradient terms. J. Diff. Equ. 252, 886-914 (2012).

[3] P. Baras and M. Pierre. Singularités éliminables pour des équations semilinéaires, Ann. Inst. Fourier (Grenoble) 34, 117-135 (1986).

[4] C. Bandle and E. Giarusso. Boundary blow-up for semilinear equations with nonlinear gradient terms, Adv. Diff. Equ. 1, 133-150 (1996).

[5] M.F. Bidaut-Véron. Liouville results and asymptotics of solutions of a quasilinear elliptic equation with supercritical source gradient term, $A d v$. Nonlinear Stud. 21, 57-76 (2021).

[6] M.F. Bidaut-Véron, M. Garcia-Huidobro and L. Véron. Boundary singular solutions of a class of equations with mixed absorption-reaction, submitted. arXiv:2007.16097v2.

[7] M.F. Bidaut-Véron, M. Garcia-Huidobro and L. Véron. Estimates of solutions of elliptic equations with a source reaction term involving the product of the function and its gradient, Duke Math. J. 189, 1487-1537 (2019).

[8] M.F. Bidaut-Véron, M. Garcia-Huidobro and L. Véron. Singular solutions of some elliptic equations involving mixed absorption-reaction, in preparation.

[9] M.F. Bidaut-Véron, Q. H. Nguyen and L. Véron. Quasilinear elliptic equations with a source reaction term involving the function and its gradient and measure data, Calc. Var. Partial Diff. Equ. 59 (5), Paper No. 148, 38 p. (2020).

[10] L. Boccardo, F. Murat and J. P. Puel. Résultats d'existence pour certains problèmes elliptiques quasilinéaires, Annali della Scuola Normale Superiore di Pisa - Classe di Scienze, Ser. 4, T. 11, 213-235 (1984).

[11] H. Brezis. Analyse fonctionnelle et Applications (Theory and Applications), Collect. Math. Appl. Maîtrise, Masson, Paris, xiv+234 pp (1983).

[12] H. Brezis and W. A. Strauss. Semilinear elliptic equation in $L^{1}$. J. Math. Soc. Japan 25, 564-590 (1973) 
[13] H. Brezis and L. Véron. Removable Singularities for Some Nonlinear Elliptic Equations. Arch. Rat. Mech. Anal. 75, 1-6 (1980)

[14] D. Gilbarg and N. S. Trudinger. Elliptic Partial Differential Equations of Second Order, reprint of the 1998 edition, Classics in Math. SpringerVerlag, New York (2001).

[15] P. Felmer, A. Quass and B. Sirakov. Solvability of nonlinear elliptic equations with gradient terms, J. Diff. Equ. 254, 4327-4346 (2013).

[16] K. Hansson, V. G. Maz'ya and I. E. Verbitsky. Criteria of solvability for multidimensional Riccati equations, Ark. Mat. 37, 87120 (1999).

[17] J. B. Keller. On the solutions of $\Delta u=f(u)$, Comm. Pure Appl. Math. 10, 503-510 (1957).

[18] P. L. Lions. Quelques remarques sur les problèmes elliptiques quasilinéaires du second ordre, J. Analyse Mathématique. 45, 234-254 (1985).

[19] V. G. Maz'ya and I. Verbitsky. Capacitary inequalities for fractional integrals, with applications to partial differential equations and Sobolev multipliers, Ark. Mat. 33, 81-115 (1995).

[20] P. T. Nguyen. Isolated singularities of positive solutions of elliptic equations with weighted gradient term, Anal. \&5 PDE 9, 1671-1692 (2016).

[21] Q. H. Nguyen and N. C. Phuc. Good $\lambda$ and Muckenhoupt-Wheeden type bounds in quasilinear measure datum problems, with applications, Math. Annalen 374 67-98 (2019).

[22] R. Osserman. On the inequality $\Delta u \geq f(u)$, Pacific J. Math. 7, 1641-1647 (1957).

[23] N.C. Phuc. Nonlinear Muckenhoupt-Wheeden type bounds on Reifenberg flat domains, with applications to quasilinear Riccati type equations, Adv. Math. 250, 387-419 (2014).

[24] N. C. Phuc and I. Verbitsky. Quasilinear and Hessian equations of LaneEmden type, Annals Math. 168, 859-914 (2008).

[25] J. Serrin. Isolated singularities of solutions of quasilinear equations, Acta Mathematica 34, 247-302 (1964).

[26] E. M. Stein. Singular Integrals and Differentiability Properties of Functions, Princeton University Press, Princeton, New Jersey (1970).

[27] I. Verbitsky and R. L. Wheeden. Weighted norm inequalities for integral operators, Trans. Amer. Math. Soc. 350, 3371-3391 (1998).

[28] L. Véron. Singular solutions of some nonlinear elliptic equations, Nonlinear Anal. Theory, Methods Appl. 5, 225-242 (1981). 